Climate Change Expert Group

Paper No. 2009(3)

\title{
Sectoral Approaches and the Carbon Market
}

R. Baron, B. Buchner (IEA)

and J. Ellis (OECD) 


\section{OECD/IEA CLIMATE CHANGE EXPERT GROUP PAPERS}

This series is designed to make available to a wider readership selected papers on climate change issues that have been prepared for the OECD/IEA Climate Change Expert Group (CCXG). The CCXG (formerly called the Annex I Expert Group) is a group of government delegates from OECD and other industrialised countries. The aim of the group is to promote dialogue and enhance understanding on technical issues in the international climate change negotiations. CCXG papers are developed in consultation with experts from a wide range of developed and developing countries, including those participating in CCXG Global Forums.

The full papers are generally available only in English.

The opinions expressed in these papers are the sole responsibility of the author(s) and do not necessarily reflect the views of the OECD, the IEA or their member countries, or the endorsement of any approach described herein.

Comments on the series are welcome, and should be sent to env.contact@oecd.org or the Environment Directorate, 2 rue André Pascal, 75775 PARIS CEDEX 16, France.

OECD/IEA Climate Change Expert Group Papers are published on www.oecd.org/env/cc/ccxg.htm

Applications for permission to reproduce or translate all or part of this material should be made to: OECD Publishing, rights@oecd.org or fax 33145249930.

This document has been produced with the financial assistance of the European Union. The views expressed herein can in no way be taken to reflect the official opinion of the European Union.

Copyright OECD/IEA, 2009

Applications for permission to reproduce or translate all or part of this material should be addressed to:

Head of Publications Service, OECD/IEA

2 rue André Pascal, 75775 Paris Cedex 16, France

or

9 rue de la Fédération, 75739 Paris Cedex 15, France. 


\begin{abstract}
Sectoral approaches are proposed as a means to broaden the global scope of greenhouse gas (GHG) mitigation to developing countries. Market mechanisms are put forward in that context to create incentives for mitigation in developing countries beyond the existing Clean Development Mechanism (CDM), and to encourage mitigation at least possible cost. The introduction of new, sector-based, market mechanisms is only one of many proposals discussed by UNFCCC Parties in the context of a post-2012 international climate policy framework, as a possible means to support mitigation actions in developing countries.

This paper considers the carbon market aspects of sectoral approaches to reduce greenhouse gas (GHG) emissions in developing countries. It discusses three general ways to link sectoral goals with the carbon market: (i) intensity goals, based on a GHG performance per unit of output; (ii) fixed emission goals, with an ex-post issuance of credits or trading with an ex-ante allocation of allowances; and (iii) technologybased sectoral objectives.

This paper explores the domestic policy implications of moving from a single project approach (i.e., CDM), to a multi-plant, sector-wide carbon market mechanism implied by sectoral crediting and trading. It also touches on possible transition issues, especially from intensity-based emission goals to fixed ones. The paper concludes that sector-based market mechanisms, regardless of the design option chosen, will require some significant upfront effort both nationally and internationally to set appropriate baselines and ensure adequate measurement, reporting and verification in order to generate economically valuable and environmentally-credible credits. Technology diffusion goals may be supported by other means than the carbon market if developing GHG baselines for such activities were too difficult. Sectoral approaches also imply some significant policy effort in countries that adhere to them, to ensure that the baselines are exceeded so that carbon market revenues are generated, and that these revenues represent effective incentives for entities to pursue GHG mitigation, wherever it is most cost-effective to do so.
\end{abstract}

JEL Classification: F53, Q54, Q56, Q58

Keywords: Climate change; mitigation; greenhouse gas; carbon market, sectoral approach 


\section{RÉSUMÉ}

Des approches sectorielles sont proposées afin d'élargir les possibilités d'atténuation des gaz à effet de serre (GES) à l'échelle mondiale en y associant les pays en développement. Les mécanismes du marché sont mis en avant, dans cette optique, pour créer d'autres incitations à l'atténuation dans les pays en développement, en plus de celles du mécanisme pour un développement propre (MDP) existant ; il est suggéré aussi d'encourager l'atténuation au moindre coût possible. L'adoption de nouveaux mécanismes du marché de portée sectorielle n'est qu'une proposition parmi beaucoup d'autres que les Parties à la CCNUCC ont examinées en prévision du cadre international dans lequel s'inscrira la politique climatique après 2012 : leur mise en œuvre pourrait faciliter les actions d'atténuation dans les pays en développement.

Ce document aborde les aspects qui se rapportent au marché du carbone dans les approches sectorielles visant à réduire les émissions de gaz à effet de serre (GES) dans les pays en développement. Il analyse trois moyens de relier, de façon générale, les objectifs sectoriels et le marché du carbone : (i) des objectifs d'intensité, fondés sur le bilan de GES par unité de production ; (ii) des objectifs d'émission fixes, assortis de la délivrance ex post de crédits ou de l'échange de quotas alloués ex ante ; et (iii) des objectifs sectoriels en fonction des technologies.

Ce document étudie les conséquences, pour les politiques nationales, du passage d'une approche axée sur des projets pris isolément (par exemple, dans le cadre du MDP) à un marché du carbone incluant de nombreuses installations et dont le périmètre est déterminé par l'attribution de crédits et la réalisation d'échanges au niveau sectoriel. Sont examinés aussi les problèmes de transition qui pourraient surgir, en particulier si des objectifs d'émission fixes remplacent les objectifs basés sur l'intensité. Ce document conclut que la mise en place de mécanismes de marché sectoriels nécessitera, quel que soit l'agencement choisi, certains travaux importants au départ, à l'échelon national aussi bien qu'international, pour définir les niveaux de référence appropriés et veiller à l'adéquation des procédures de mesure, de notification et de vérification, en vue de générer des crédits économiquement avantageux et crédibles sur le plan environnemental. Si la mise au point des niveaux de référence des GES pour les activités du marché du carbone pose trop de difficultés, d'autres moyens peuvent favoriser la réalisation des objectifs de diffusion des technologies. Par ailleurs, les autorités des pays qui adhèreront aux approches sectorielles devront s'assurer, par un important effort, que les niveaux de référence pourront être dépassés, afin que le marché du carbone dégage des recettes qui représentent de réelles incitations à l'atténuation des GES, chaque fois que ce sera la solution la plus efficace par rapport aux coûts.

Classification JEL: F53, Q54, Q56, Q58

Mots-clés: Changement climatique; atténuation; gaz à effet de serre; marchés du carbone; approche sectorielle 


\section{FOREWORD}

This document was prepared by the OECD and IEA Secretariats in Spring 2009 in response to the Annex I Expert Group on the United Nations Framework Convention on Climate Change (UNFCCC). The Annex I Expert Group oversees development of analytical papers for the purpose of providing useful and timely input to the climate change negotiations. These papers may also be useful to national policy-makers and other decision-makers. In a collaborative effort, authors work with the Annex I Expert Group to develop these papers. However, the papers do not necessarily represent the views of the OECD or the IEA, nor are they intended to prejudge the views of countries participating in the Annex I Expert Group. Rather, they are Secretariat information papers intended to inform Member countries, as well as the UNFCCC audience.

The Annex I Parties or countries referred to in this document are those listed in Annex I of the UNFCCC (as amended at the 3rd Conference of the Parties in December 1997): Australia, Austria, Belarus, Belgium, Bulgaria, Canada, Croatia, Czech Republic, Denmark, the European Community, Estonia, Finland, France, Germany, Greece, Hungary, Iceland, Ireland, Italy, Japan, Latvia, Liechtenstein, Lithuania, Luxembourg, Monaco, Netherlands, New Zealand, Norway, Poland, Portugal, Romania, Russian Federation, Slovakia, Slovenia, Spain, Sweden, Switzerland, Turkey, Ukraine, United Kingdom of Great Britain and Northern Ireland, and United States of America. Korea and Mexico, as OECD member countries, also participate in the Annex I Expert Group. Where this document refers to "countries" or "governments", it is also intended to include "regional economic organisations", if appropriate.

\section{ACKNOWLEDGEMENTS}

This paper was prepared by Richard Baron, Barbara Buchner (IEA) and Jane Ellis (OECD). The authors would like to thank Rick Bradley, André Aasrud, Keisuke Inoue, Philippine de T’Serclaes (IEA), Helen Mountford, Christa Clapp, Katia Karousakis, Cuauhtemoc Rebolledo-Gómez (OECD) for their help and comments on the paper, Cédric Philibert (IEA) for earlier discussions on this topic, as well as Lambert Schneider (Öko-Institut), Emmanuel Fages (SG orbeo), Steven Gray (Climate Change Capital), Mark Lewis (Deutsche Bank), Sophie Galharret, and Emmanuel Guérin (Iddri) for the information, comments and ideas they provided. The authors are also indebted to the delegates of the Annex I Expert Group on the UNFCCC for their useful comments and guidance.

\section{Questions and comments should be sent to:}

Christina Hood

IEA

9 rue de la Fédération

75739 Paris Cedex 16

France

Email: christina.hood@iea.org
Jane Ellis

OECD Environment Directorate

2 rue André-Pascal

757775 Paris Cedex 15

France

Email: jane.ellis@oecd.org

All OECD and IEA information papers for the Annex I Expert Group on the UNFCCC can be downloaded from: www.oecd.org/env/cc/ccxg.htm. 


\section{TABLE OF CONTENTS}

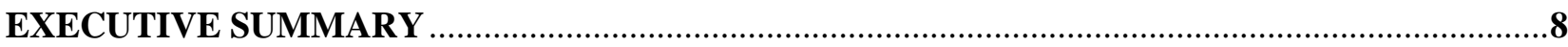

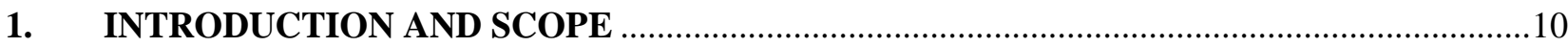

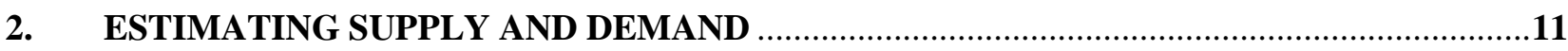

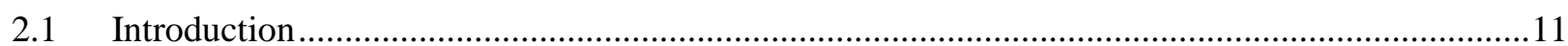

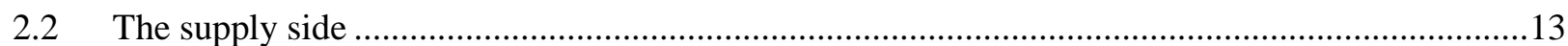

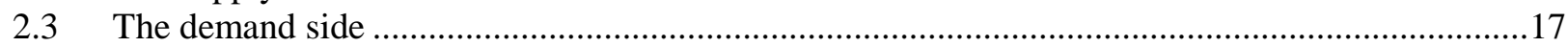

3. HOW WOULD SECTORAL MARKET MECHANISMS WORK? ........................................22

3.1 Implementing sectoral crediting based on intensity goals ..........................................................22

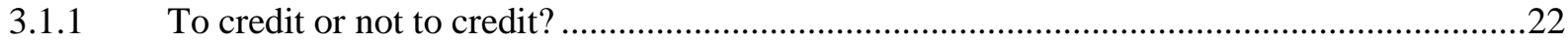

3.1.2 Domestic implementation scenarios and link to international market..................................24

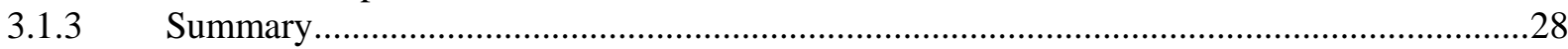

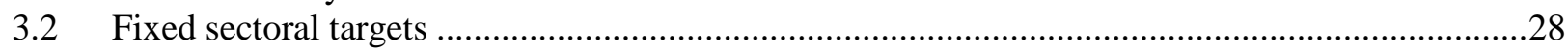

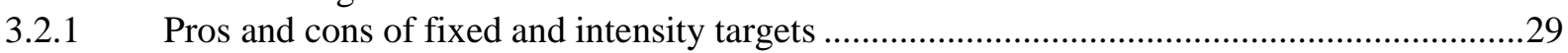

3.2.2 Sectoral crediting mechanisms with "no lose" fixed baselines ..........................................30

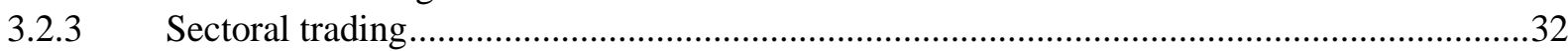

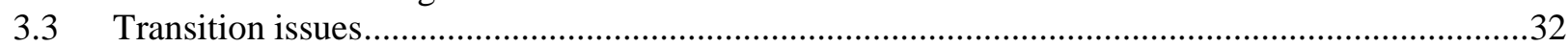

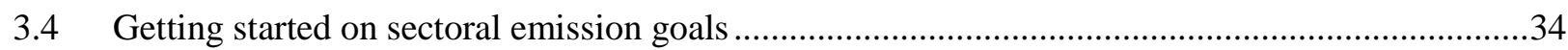

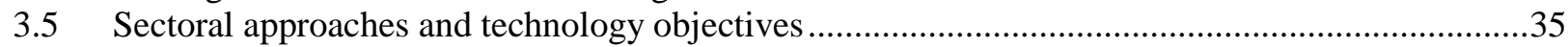

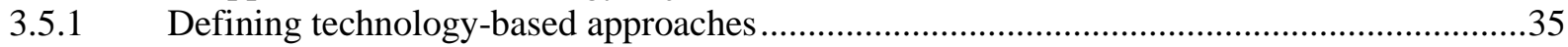

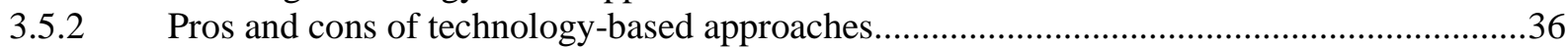

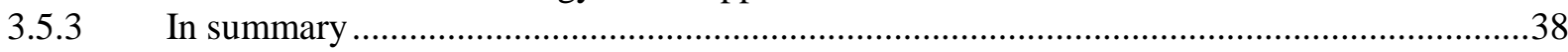

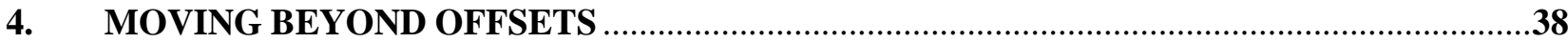

4.1 An economic assessment of restrictions on the supply of GHG credits ......................................39

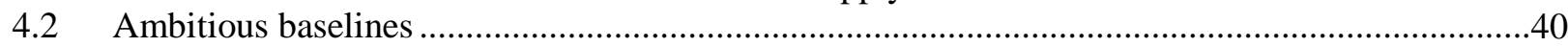

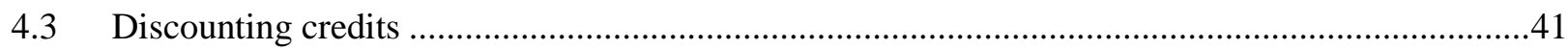

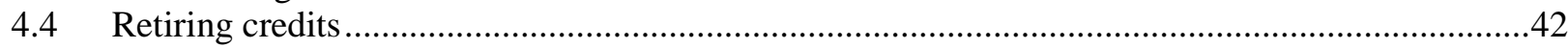

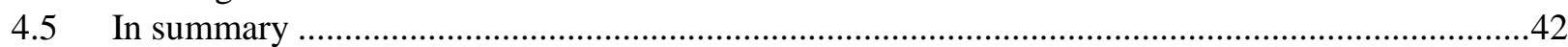

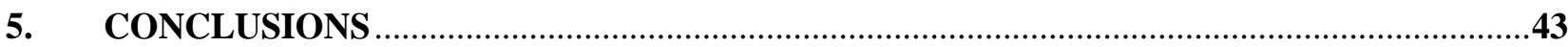

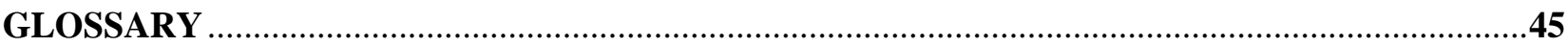

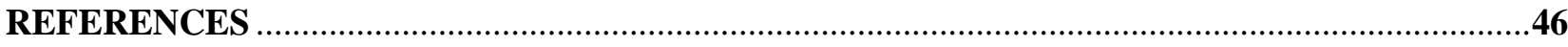

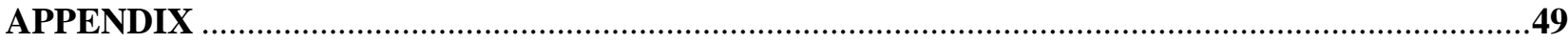




\section{LIST OF TABLES}

Table 1: $\quad$ Annual mitigation potential in major emerging economies in selected sectors ...................... 13

Table 2: $\quad$ Annual mitigation and or crediting potential during 2013-2020 - Selected sectors

(in $\mathrm{MtCO}_{2} \mathrm{eq}$ )

Table 3: Sectoral crediting illustration: revenues to government........................................................... 49

Table 4: $\quad$ Enhancing crediting certainty using a performance reserve .................................................... 49

\section{LIST OF FIGURES}

Figure 1: $\quad$ Energy-related $\mathrm{CO}_{2}$ emissions in 2020 under business-as-usual 12

Figure 2: $\quad$ Summarising potential annual post-2012 demand and supply for credits in selected sectors $\left(\mathrm{MtCO}_{2} \mathrm{eq}\right)$

Figure 3: Crediting under a "no lose” sectoral target ............................................................................. 24

Figure 4: $\quad$ Sectoral crediting option - Credits to sources in compliance .............................................. 26

Figure 5: $\quad$ Securing credits with a performance reserve ...................................................................... 27

Figure 6: Government assumes liability for any overselling.............................................................. 27

Figure 7: "No lose" sectoral crediting mechanism (with fixed baseline) ............................................ 31

Figure 8: No lose” sectoral crediting mechanism with a fixed baseline............................................ 31

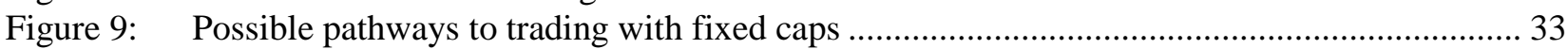

Figure 10: The carbon market without limits on CDM use................................................................ 40 


\section{Executive Summary}

Sectoral approaches are proposed as a means to broaden the global scope of greenhouse gas (GHG) mitigation to developing countries. Market mechanisms are put forward in that context to create incentives for mitigation in developing countries beyond the existing Clean Development Mechanism (CDM), and to encourage mitigation at least possible cost. The introduction of new, sector-based, market mechanisms is only one of many proposals discussed by UNFCCC Parties in the context of a post-2012 international climate policy framework, as a possible means to support mitigation actions in developing countries. The role of such sectoral mechanisms will eventually be determined by the emission goals that accompany them, and on how the Parties decide to harness the carbon market and other mechanisms to support mitigation in developing countries. These aspects are also currently under negotiation.

This paper considers the carbon market aspects of sectoral approaches to reduce greenhouse gas (GHG) emissions in developing countries. Three general ways to link sectoral goals with the carbon market are discussed:

- Intensity goals - based on a GHG performance per unit of output.

- Fixed emission goals - an absolute total quantity of GHG emissions - as the basis for crediting, with an ex post issuance of credits, or trading, with an ex ante allocation of allowances.

- Technology-based sectoral objectives.

With the market at the core of the effectiveness of crediting mechanisms, proposals for such mechanisms must be put in the context of potential supply and demand projections. The paper thus presents orders of magnitude relevant to this question. Estimates are preliminary, given the absence of more defined approaches and signals from countries on the acceptability of sectoral market mechanisms. These estimates of the potential credit supply for the power, cement and forestry sectors alone are much larger than current estimates of possible demand by the European Union and the United States of America. Assuming that CDM would continue in other sectors, this information calls for a careful assessment of sectoral options for crediting to avoid raising unrealistic expectations on possible finance from the carbon market. Parties will need to decide on sectors that are amenable to sectoral market mechanisms. Moving towards implementation, important choices would also need to be made on which entities to include (based on size of operations, age of plants, etc.). These choices would influence the role and the effectiveness of these mechanisms.

Broadening CDM-type crediting to whole sectors has advantages and disadvantages. It raises concerns about the offsetting nature of generated credits - the case now under CDM: the Mechanism helps to lower the cost of mitigation action, but does not contribute to global emission reductions. Various proposals have been tabled to evolve beyond offsetting. Moving from project-based crediting to sector-based crediting or trading implies a negotiation over the baseline (or target) level, the level of "unilateral" action by developing countries, and on additional support to implement policies and to facilitate the acquisition of technologies. Ambitious baselines may be sufficient to go beyond offsetting, but other options could also play a role. These could include the retirement or the discounting of Certified Emission Reductions CERs (at the supply side, or by the buyers, requiring more than one unit to cover one $\mathrm{tCO}_{2}$-eq.).

This paper explores the domestic policy implications of moving from a single project approach (i.e., CDM), to a multi-plant, sector-wide carbon market mechanism implied by sectoral crediting and trading. With sectoral crediting, i.e. an ex post issuance of credits to the country, we find that the carbon market incentive to individual investors in mitigation may be less direct, and therefore weaker than that under a single project configuration like the CDM. Under sector-wide crediting, an entity's good performance can be offset by the lack of progress of other entities in the sector, resulting in low or even zero crediting to the sector as a whole; the entity's efforts could not be fully rewarded by the carbon market in such cases. 
Solving this problem would require the government either to fine bad performance and reward good performance with the revenues, or to guarantee credits to entities that outperform their goals and take international liability if the country goal were not met. Other reasons for the less direct market signal include possible decisions by domestic governments on whether and how they would use revenues from such a mechanism, and on policy measures they would implement to trigger improvements in the sectors covered.

Fixed emission goals offer more potential to provide a direct carbon price signal to domestic entities, provided governments are willing and able to allocate efforts, i.e. devolve targets to entities. Trading with ex ante allocation of allowances and the possibility of participating in the carbon market (once allocations have been agreed) would also be preferable to crediting, which generates ex post credits. Crediting introduces uncertainty on the total quantity of credits that a country could transfer (although this could be reduced to some extent by issuing credits on an annual basis as opposed to an issuance at the end of a multi-year period). Trading requires, however, that the government takes full liability in case of noncompliance, a commitment that is of a different nature than a "no lose" crediting mechanism.

Technology-based objectives have recently raised the interest of some Parties, as a basis for implementing sectoral approaches and generating access to international support. A country may indeed decide to implement a technology diffusion approach domestically as its primary means to reach, and/or outperform, a sectoral emissions baseline (intensity or fixed). A country could also seek funding on the basis of a technology penetration goal. In the absence of a pre-agreed GHG baseline, how such an objective could lead to GHG crediting would require further research.

The paper also touches on possible transition issues, especially from intensity-based emission goals to fixed ones. Would an intensity-based mechanism hinder a transition to fixed emission goals? Due to the range of policy tools that governments could adopt to implement sectoral intensity goals, and their detailed design parameters, it is difficult to answer this question fully. It may be useful to revisit this question from the angle of the building blocks of a trading mechanism based on fixed emissions, and explore what policy measures could be undertaken early to create those.

It is clear that sector-based market mechanisms, regardless of the design option chosen, will require some significant upfront effort both nationally and internationally to set appropriate baselines, ensure adequate measurement, reporting and verification, to generate economically valuable and environmentally-credible credits. Technology diffusion goals may be supported by other means than the carbon market if developing GHG baselines for such activities were too difficult. Sectoral approaches also imply some significant policy effort in countries that adhere to them, to ensure that the baselines are exceeded so that carbon market revenues are generated, and that these revenues represent effective incentives for entities to pursue GHG mitigation, wherever it is most cost-effective to do so. 


\section{Introduction and Scope}

As outlined elsewhere (e.g. Baron et al., 2008) there are different possible sectoral approaches that could be taken forward in the post-2012 climate regime. Some of these approaches would generate credits, and therefore have an impact on the international carbon market. Recent modelling by the OECD shows that carving out sectors from general trading would lead to lower economic efficiency and higher overall cost of mitigation. It seems reasonable to assume that if crediting were to take place at sector level, Parties would wish to integrate sector-based market mechanisms in other trading instruments (OECD, 2008).

Proposals on sectoral approaches that rely on the carbon market to foster emission reductions include: ${ }^{1}$

1. A sectoral crediting mechanism (SCM) which would credit emission reductions in a sector against a defined no-lose baseline. The baseline could be set on an intensity basis, or as a fixed quantity of emissions. Such a mechanism has been proposed as sectoral CDM or as sectoral no-lose targets in the negotiations on flexible mechanisms in a future climate regime.

2. Sectoral trading, based on fixed targets, with an ex ante allocation.

3. Technology diffusion objectives.

Other policy options have been proposed, e.g. crediting for nationally-appropriate mitigation actions. There may not be substantial differences between this option and those listed above. In the end, how effective these instruments will be depends on how the price signal from the carbon market is passed on to individual sources, i.e. the value of carbon, and how these sources react to it.

These options, as tabled by various Parties and organisations, can overlap in part, but also differ in several respects, from the process they would require under the UNFCCC, to the eligibility of countries for participation in each. For some options, finance from the sale of credits through the carbon market would constitute the main form of support, while others envision first other forms of financial support to foster mitigation and crediting as an add-on.

The assumption in this paper is that sectoral crediting would generate credits ex post, for reductions achieved at the sectoral level, below a pre-established baseline. Sectoral trading, based on fixed sectoral targets, is similar to cap-and-trade and would involve the allocation of allowances ex ante. Technology diffusion objectives could also form the basis of sectoral goals, and as such open the question of crediting in case of over-compliance.

Earlier work (Baron et al., 2008; Schneider, 2008) have pointed out the role of the carbon market and various policy designs in the discussion of sectoral approaches. This paper illustrates how sector-based market mechanisms could function as a means to foster mitigation in developing countries. It focuses on three dimensions:

- Section 2 considers the volume of credits that such mechanisms could generate, given sectoral emission trends and mitigation potentials.

- Section 3 explores how the carbon market would interface with various types of sector-wide objectives in countries, as the basis for crediting or trading. What are some of the practical implementation questions that ought to be addressed by countries engaging in such policy?

- Section 4 examines how sectoral crediting systems could be designed to enhance global GHG mitigation, i.e. to go beyond the current role of CDM's CERs as one-for-one offsets for emissions in developed countries.

\footnotetext{
1 See Czech Republic , 28 April 2009, “A negotiation text for consideration at AWG-LCA 6”, and also France on behalf of the European Community and its Member States, 5 November 2008: "Analysis of means to reach emission reduction targets and identification of ways to enhance their effectiveness and contribution to sustainable development: emissions trading and the project-based mechanisms (AWG-KP). Further input in relation to the elements on possible improvements to emissions trading and the project-based mechanisms under the Kyoto Protocol”. See also Japan's Submission on Application of Sectoral Approaches, COP14, Poznan.
} 


\section{Estimating Supply and Demand}

\subsection{Introduction}

The magnitude of future credit supply is at the centre of discussions on sector-based market mechanisms. These mechanisms aim at creating a financial incentive for countries/installations to improve their emissions performance. A large potential for generating credits without a corresponding demand could reduce the carbon price and discourage mitigation in countries that may have expected significant financial support from a crediting mechanism. Unless there is a possibility for full time flexibility and banking, a low price would also have detrimental implications on investment in the development and deployment of low-carbon technologies (in developed and developing economies); it would prolong GHG-intensive practices, making it more expensive to cut emissions in the future.

Projections of supply and demand for sector-based credits are plagued with uncertainty, as explained below. This section does not attempt to address all underlying questions, but to estimate the order of magnitude of the supply that sectoral crediting mechanisms could generate, and of demand based on current policy announcements. To avoid adding uncertainty, these estimates focus on 2013-2020, a relevant time horizon for an instrument that may come into play after the Kyoto Protocol's first commitment period. The analysis below relies on existing scenarios, policy proposals and published estimates from carbon market analysts and observers.

The following orders of magnitude are useful to put these estimates in perspective: global GHG emissions from all sources amounted to $47 \mathrm{GtCO}_{2} \mathrm{eq}$ in 2005, and are expected to increase to $53 \mathrm{GtCO}_{2}$ eq by 2020 under a business-as-usual scenario (OECD, 2008). ${ }^{2}$ Global energy-related $\mathrm{CO}_{2}$ emissions are expected to constitute the main share, reaching $36.4 \mathrm{GtCO}_{2} \mathrm{eq}$ in 2020 , and are driven by the power sector $(44 \%)$ and industry (16\%) (IEA, 2008). Almost half of the 2020 energy-related $\mathrm{CO}_{2}$ emissions are expected to be emitted by the major emerging economies ${ }^{3}$, where the power sector $(51 \%)$ and industry $(21 \%)$ play an even larger role.

Against this background, the importance of a few regions, e.g. the OECD countries as well as the major emerging economies, and of a few sectors becomes clear. In addition to electricity and industry, forestry is both a major source and sink of GHG emissions. These regions and sectors are expected to significantly influence the carbon market, should sectoral crediting or trading mechanisms be recognised under a post2012 climate change regime.

\footnotetext{
2 Note that there is considerable uncertainty associated to estimates from some countries and some sectors.

3 “Major emerging economies” include China, Russia, India, Iran, Saudi Arabia, Indonesia, Brazil and South
} Africa. 
Major emerging economies

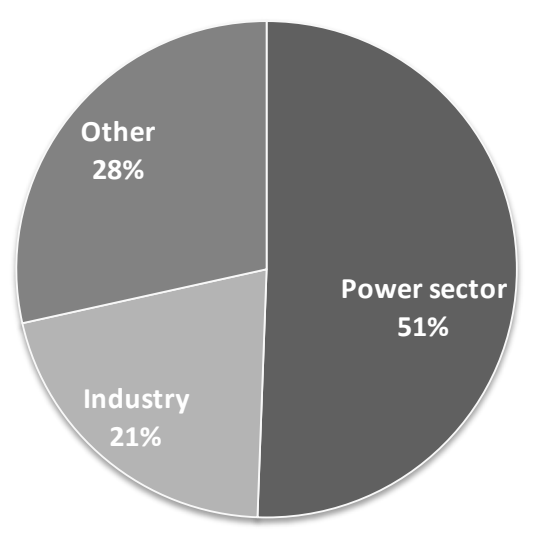

\section{Global}

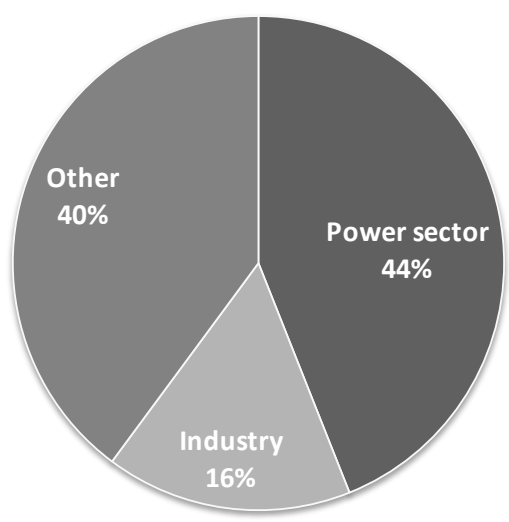

Source: World Energy Outlook 2008 (IEA, 2008)

Note: “Major emerging economies” include China, Russia, India, Iran, Saudi Arabia, Indonesia, Brazil and South Africa.

Estimates of 2013-2020 supply and demand of credits are highly uncertain as there are a number of unanswered questions at this stage:

- How fast will developing countries, and specifically the sectors in consideration, grow in the near future? How will energy prices evolve, and how will they drive efficiency improvements?

- How will international climate policy develop, i.e. what are possible commitments by countries and attitudes towards crediting?

- How will mitigation policies affect GHG emissions? How will they influence the demand for electricity and other commodities?

- Which sectors would be covered by a sectoral crediting mechanism (SCM) or sectoral trading, and which countries would participate? How quickly could countries become eligible to participate in such mechanisms?

- How ambitious would baselines (in the case of intensity-based targets) or fixed targets be?

What incentives would a sectoral mechanism create for the countries to further reduce GHG emissions in the sector? Would the countries meet the sectoral baselines or targets and, if yes, to what extent would they manage to exceed them?

It is also important to recall that currently two major carbon markets co-exist: one carbon market established for compliance of governments with the Kyoto Protocol, and a second carbon market, essentially the EU ETS, with restricted access to the international market ${ }^{4}$. In essence, governments and non-EU entities have the right to access all Kyoto units, while the EU ETS entities do not.

Other organisations have also estimated supply and demand for GHG units post-2012. The Carbon Trust (2009) first estimates the supply and demand situation until 2012, and projects a total supply of 2,050$4,000 \mathrm{MtCO}_{2}$ from international carbon mechanisms, largely in excess of the potential demand of 890-

4 There is a third carbon market, i.e. the voluntary market. This is much smaller, but is growing rapidly. 
$1,830 \mathrm{MtCO}_{2}$ by governments and companies in the EU ETS. The analysis assumes that governments would use to a certain extent AAUs through Green Investment Schemes (GIS) but that most AAUs would be banked for use post-2012. This, together with an extrapolation of today's inflow of CDM and JI projects, and the existence of surplus AAUs in the Russian Federation and Ukraine in particular, leads to an estimated supply of $19.5 \mathrm{GtCO}_{2}$ in the period 2013-20. These calculations extrapolate existing mechanisms, i.e. do not consider possible sector-based market mechanisms.

The next sections explore first the possible supply from sector-based mechanism, and second, potential demand. We should recall, however, that the Kyoto Protocol mechanisms in the first commitment period promise a fairly large excess supply of compliance units, although they may not be eligible in all demand markets, if today's practice is any indication.

\subsection{The supply side}

We focus here on the possible supply of credits from sectoral market mechanisms applied in developing countries. To obtain a reasonable estimate, it is useful to consider the orders of magnitude of emission reductions achieved in these countries under various climate policy scenarios (Table 1).

Table 1: Annual mitigation potential in major emerging economies in selected sectors

\begin{tabular}{l|c|c|c|c|c|c}
\hline Source & $\begin{array}{c}\text { Policy } \\
\text { scenario }\end{array}$ & GHG coverage & Electricity & Industry & Transport & Forestry \\
\hline IEA $(2008)^{1}$ & $\begin{array}{c}450 / 550 \\
\text { stabilisation }\end{array}$ & $\begin{array}{c}\text { Energy-related } \\
\text { CO }_{2}\end{array}$ & 775 & 310 & 38 & - \\
\hline $\begin{array}{l}\text { OECD } \\
(2009)^{2}\end{array}$ & $\begin{array}{c}\text { Tax 20 (USD } \\
2005)\end{array}$ & $\begin{array}{c}\text { All GHG } \\
\text { emissions }\end{array}$ & 3114 & 970 & 110 & $?$ \\
\hline
\end{tabular}

All: 2013-2020, in $\mathrm{MtCO}_{2} \mathrm{eq}$

Note: In IEA (2008), the 450 and 550 ppm scenarios follow the same path until 2020. The OECD model is a general equilibrium macro-economic model, which generally shows higher response to price increases. This partly explains the high level of mitigation recorded for a USD20/tCO 2 .

${ }^{1}$ In IEA(2008), “major emerging economies” include China, Russia, India, Iran, Saudi Arabia, Indonesia, Brazil and South Africa.

${ }^{2}$ This model includes China, Russia, India, Indonesia, Brazil, and South Africa as the "major emerging economies". These results are drawn from analysis using the OECD ENV-Linkages model (Karousakis, personal communication). The Tax 20 scenario is comparable to the IEA trajectory in 2020, as its $\mathrm{CO}_{2}$ emissions in 2020 amount to 30.9 $\mathrm{GtCO}_{2}$ eq while the IEA 450/550 stabilisation scenario leads to $31.6 \mathrm{GtCO}_{2}$ eq of energy related $\mathrm{CO}_{2}$ emissions.

The mitigation potentials shown above would generally be higher than the potential amount that would be supplied to the carbon market. For example, in the power sector, if a sectoral crediting mechanism is based on an intensity target, the volume of credits is commensurate with electricity output. Thus, with an identical performance under the baseline, in terms of $\mathrm{CO}_{2}$ intensity, a lower demand for electricity driven by end-use efficiency improvements would lower the credits generated. ${ }^{5}$ We deduct this mitigation potential from the total over which crediting could apply at the generation side. Applying this method to the IEA $450 \mathrm{ppm}$ policy scenario, we estimate that that a sectoral crediting mechanism in the power sector could account for up to $465 \mathrm{MtCO}_{2}$ annually (3.7 $\mathrm{GtCO}_{2}$ over the period 2013-2020), which reflects total mitigation potential. This is a maximum however, as an ambitious baseline (e.g. as envisaged by the EU under "no lose" approaches) would adjust this level downwards.

If the targets were fixed rather than intensity-based, surplus allowances for sale would be generated for any reduction below the target. With targets set at the business-as-usual level, this approach would result in a

5 IEA simulations considering a 450 ppm objective (for all gases) estimate that end-use energy efficiency gains account for more than $50 \%$ of the emission mitigation potential, and specifically about $40 \%$ for electricity demand (IEA, 2008). 
higher supply of units for sale, e.g. the whole $775 \mathrm{MtCO}_{2}$ in the IEA scenario, by 2020, and $3 \mathrm{GtCO}_{2}$ for the OECD scenario. These numbers appear unlikely, as any fixed targets that would be agreed are likely to reflect some environmental ambition, and therefore would be set lower than business-as-usual.

Recent studies provide more information on expected credit flows for power generation, some heavy industry and forestry in particular:

- Power generation. To our knowledge, three studies analyse the credit flows of a sectoral crediting mechanism in the power sector. ${ }^{6}$

Amatayakul et al. (2008) evaluate a voluntary scheme that would allow developing countries with high $\mathrm{CO}_{2}$ emissions from electricity generation, including China and India, ${ }^{7}$ to earn carbon credits if emissions in the power sector are below a specific emissions intensity target that declines annually. Baselines are drawn on a country-by-country basis. Depending on the policy scenario, the overall mitigation potential is estimated between 1.8 and $5.7 \mathrm{GtCO}_{2}$ between 2013 and 2020, and after imposing an intensity target and an baseline scenarios varying in stringency (building from existing CDM methodologies), a total amount of ca. 0.9 to $4.5 \mathrm{GtCO}_{2}$ could be credited or between $110 \mathrm{Mt}$ and $560 \mathrm{Mt}$ annually.

GWEC (2008) uses different carbon price assumptions to highlight potential carbon revenues for a number of different sectoral no-lose targets in the Chinese power sector. Roughly in line with Amatayakul et al. (2008), but obtaining much larger upper range numbers, they estimate that the maximum quantity of average annual emission reduction credits between 2010- 2020 could range between 154 and $767 \mathrm{MtCO}_{2}$ - for China only. Using carbon price in the EUR15-50/tCO 2 range, this would represent annual carbon revenues between EUR2.3 and EUR38 billion.

Schmidt et al. (2008) analyse a system in which the ten highest-emitting developing countries pledge to achieve "no lose" emissions intensity targets in the power sector - a 3\% reduction per year, while energy efficiency improvements reduce growth in production by $0.5 \%$ annually. If achieved, this objective could reduce GHG emissions by $33 \%$ in 2020, or $1.3 \mathrm{GtCO}_{2} \mathrm{eq}$. If crediting occurred on actual electricity output, it would apply to roughly $1.17 \mathrm{GtCO}_{2} \mathrm{eq}$. The authors do not indentify a baseline, however.

- In heavy industry, the cement sector is the single largest contributor to GHG emissions. In 2007, it was responsible for over $2 \mathrm{GtCO}_{2}$, representing over eight percent of global $\mathrm{CO}_{2}$ emissions. Based on different data sources including domestic analyses and technology information (IEA, 2008b), CCAP et al (2008) identify a mitigation potential of $460 \mathrm{MtCO}_{2}$ in 2020 in the cement sector in China, Mexico and Brazil under the most advanced "no lose" target. ${ }^{8}$ Note, however, that this again represents the possible total of emission reductions and not the volume of credits - a baseline would be needed for this, different from the business-as-usual scenario.

Schmidt et al. (2008) also consider a "no lose" sectoral target for the cement sector, assuming that developing countries with highest $\mathrm{CO}_{2}$ emissions from this sector pledge to converge towards an intensity target of 0.6 tonnes of $\mathrm{CO}_{2}$ per tonne of cement by $2020 .{ }^{9}$ This scenario would reduce

\footnotetext{
6 This question will be covered in more detail in IEA (2009).

7 The ten developing countries with the largest $\mathrm{CO}_{2}$ emissions from electricity generation have been identified, but three of them - Saudi Arabia, Iran, and Kazakhstan - were excluded from the analysis due to lack of data. China, India, South Africa, South Korea, Mexico, Indonesia and Thailand were part of the analysis.

8 The most advanced no-lose target assumes that today's best practices of best available technologies are adopted in CCAP et al (2008), resulting in potential emissions reductions in the cement sector in major developing countries of $15-18 \%$ of BAU in 2020.

9 The year 2000 average is 0.77 tonne $\mathrm{CO}_{2} /$ tonne cement.
} 
GHG emissions from cement in 2020 by $25 \%$ from the reference scenario, or $450 \mathrm{MtCO}_{2} \mathrm{eq}$. The study also evaluates a "no lose" target for the iron and steel sector, requiring the highest-emitting developing countries to converge towards a carbon intensity of steel of 0.8 tonnes of $\mathrm{CO}_{2}$ per tonne steel by $2025 .^{10}$ This sectoral improvement would reduce GHG emissions from iron and steel in 2020 by $54 \%$ from business-as-usual, or $1 \mathrm{GtCO}_{2} \mathrm{eq}$.

- Forestry. Reduced emissions from deforestation and degradation in developing countries (REDD) is an area that has received significant attention under the UNFCCC, as a sector for enhanced mitigation action post-2012. Proposals from developing countries on how to enhance REDD currently include both market-based and fund-based mechanisms to finance emission reductions. REDD credits are already being traded on the voluntary carbon market and significant efforts are underway to mobilise funds for REDD to support capacity-building in developing countries. In addition, while not necessarily requiring an immediate, complete linkage of the forestry sector with the carbon market, the idea to use the carbon market has become prominent and evolved towards various variations (see Box 1 for a recent proposal by the European Commission). ${ }^{11}$

Kindermann et al. (2008) summarise estimates of the mitigation potential in REDD, based on three global models. ${ }^{12}$ Based on this study, ONF International (2008) assume that only half of the indicated mitigation potential would be achievable between 2013-2020. They find that REDD ${ }^{13}$ may yield cumulative reductions amounting to $10.8 \mathrm{GtCO}_{2} \mathrm{e}$ over 2013 to 2020, or $1.35 \mathrm{GtCO}_{2} \mathrm{e}$ on average annually. This corresponds to $7.5 \%$ of Annex B base year emissions and may cost EUR15 billion. ${ }^{14}$ This is in line with recent results by New Carbon Finance (2009), which calculate that 1.4 $\mathrm{GtCO}_{2} \mathrm{eq}$ in 2020 can be achieved at a marginal cost of EUR10/tCO ${ }_{2}$.

Though a number of technical and methodological issues remain to be resolved before REDD could be integrated into the existing carbon market (in particular MRV issues and capacitybuilding in developing countries), these estimates illustrate a large mitigation potential. Again, how the actual supply of REDD credits compares with this would depend on which countries participate, and on how crediting baselines are established.

10 The year 2000 average is 1.53 tonne $\mathrm{CO}_{2}$ /tonne steel.

11 For a discussion of market- and non-market-based proposals to reduce emissions from deforestation and degradation in developing countries see Karousakis and Corfee-Morlot (2007).

12 A number of studies have estimated mitigation potential of reducing forest emissions (see ONF International, 2008 and Wertz-Kanounnikoff, 2008 for a review of these).

13 REDD+ covers deforestation and forest degradation, forest restoration, and forest activities leading to enhanced carbon storage.

14 Note that this study attempts to include transaction costs, costs related to asymmetry of information and other institutional barriers, and therefore indicates higher, but maybe more realistic costs of REDD than previous studies. 


\section{Box 1. The EU proposal on deforestation and forest degradation}

In October 2008, the European Commission published a communication addressing the challenges of deforestation and forest degradation to tackle climate change and biodiversity loss, proposing a two track approach under the UNFCCC: ${ }^{15}$

- The establishment of a Global Forest Carbon Mechanism (short-term response): developing countries ratifying the future UNFCCC global agreement for 2013-2020 can obtain financial support for REDD, including for capacity-building. Financial support for action by a country against deforestation and forest degradation would be performance-based (evaluated in $\mathrm{CO}_{2}$ terms) and provided ex-post on the basis of assessed, monitored and verified results.

Testing the inclusion of deforestation in carbon markets (longer-term response): in addition to the continued recognition of afforestation and reforestation activities under the CDM, the recognition of deforestation credits for government compliance is to be investigated as a longer-term response (i.e. post-2012). Several preconditions would need to be met; in particular, additionality needs to be ensured and issues related to the permanence of forestry credits and liability must be resolved. In this context, a new sectoral market mechanism could avoid leakage problems. Inclusion of forestry credits in the EU ETS should only be considered after a thorough review of the experience of using deforestation credits for government compliance and for the period after 2020.

Table 2 summarises the mitigation potentials identified in several key sectors, under different policy assumptions. These estimates could be used as reference points for a discussion and serve as a starting point to estimate the potential credit supply in sectoral crediting mechanisms.

Table 2: Annual mitigation and or crediting potential during 2013-2020 - Selected sectors (in $\mathrm{MtCO}_{2} \mathrm{eq}$ )

\begin{tabular}{|c|c|c|c|c|}
\hline Source & Regional focus & Power & Cement $^{4}$ & Forestry \\
\hline IEA (2008) & $\begin{array}{l}\text { Major emerging } \\
\text { economies }^{1}\end{array}$ & $465^{2}$ & - & - \\
\hline $\begin{array}{l}\text { Amatayakul et al. } \\
\text { (2008) }\end{array}$ & $\begin{array}{l}\text { China, India, South } \\
\text { Africa, South Korea, } \\
\text { Mexico, Indonesia, } \\
\text { Thailand }\end{array}$ & $\begin{array}{c}110-560 \\
\text { (most ambitious: } \\
484)^{3}\end{array}$ & - & - \\
\hline GWEC (2008) & China & $154-767$ & - & - \\
\hline Schmidt et al. (2008) & $\begin{array}{l}\text { Largest developing } \\
\text { country emitters }\end{array}$ & 1,170 (est.) & 450 & \\
\hline CCAP et al. (2008) & China, Mexico, Brazil & - & $460^{5}$ & - \\
\hline IEA (2008) & China, Mexico, Brazil & - & 560 & - \\
\hline Ecofys (2008) & China, Mexico, Brazil & - & 720 & - \\
\hline $\begin{array}{ll}\text { ONF } & \text { International } \\
(2008) & \\
\end{array}$ & Developing countries & - & - & 1,350 \\
\hline $\begin{array}{l}\text { New Carbon Finance } \\
(2009)\end{array}$ & Developing countries & - & - & 1,400 \\
\hline
\end{tabular}

Note: The data in this table are drawn from different studies with different assumptions. Only the numbers for the power sector represent crediting potential, while the other studies provide figures for the overall mitigation potential in the specific sector and region.

${ }^{1}$ This group includes China, Russia, India, Iran, Saudi Arabia, Indonesia, Brazil and South Africa.

${ }^{2}$ Accounting for a $40 \%$ energy efficiency improvement (the amount of electricity saved between the IEA Reference and 450/550 scenarios), this represents all mitigation in generation as a result of fuel mix changes, not from lower levels of demand. ${ }^{3}$ Assuming baseline and intensity targets of varying stringency.

${ }^{4}$ Year 2020.

${ }^{5}$ Assuming the most advanced "no lose” targets based on current best practices or best available technologies.

15 EU(COM)2008 645/FINAL. 
The table shows that estimates of the mitigation potential and credit supply diverge significantly among different studies that diverge in their assumptions on policy and on future trends. The current financial crisis or other unforeseen events can also considerably affect the supply of GHG credits. Next to the large volumes of potential credit generation, the high uncertainty of any ex ante estimate of credit supply is a key policy challenge in designing and regulating a global carbon market.

\subsection{The demand side}

Demand for credits is assumed to come from developed country Parties that adopt quantified emission targets, with the possibility to achieve these targets at a lower overall cost, given access to the international carbon market.

Estimating potential demand for credits is challenging on two grounds: first, binding emission targets (or goals) for post-2012 have not yet been announced, let alone adopted, by several developed countries. Second, there is therefore no indication of a possible compliance gap, and uncertain information on the cost curves of these countries and of sectors in developing countries, and no information on baselines. All these elements would be necessary to evaluate quantities that could be traded under unrestricted market conditions.

On the other hand, some developed countries have expressed reluctance to purchase vast amounts of international offsets for the purpose of compliance, and in some cases provided quantified limits to such purchases (see below). The general policy trend seems to be towards a restricted, not unlimited, demand for credits post-2012.

Calculations here focus on the EU and the US, as well as on indications from the international policy process. In the recent climate and energy package, ${ }^{16}$ the EU details the possible access to credits for sectors under its emissions trading system, as well as for non-EU ETS activities. Concerns about the implications of the use of credits for the European carbon market and domestic abatement figured prominently during the negotiations on the post-2012 revision of the system, recalling similarities with discussions on a global scale. Box 2 discusses the EU approach vis-à-vis credits.

The EU allows access to credits worth about 2.8 to $3.1 \mathrm{GtCO}_{2}$ over the period 2008 to 2020. Notwithstanding the current economic downturn, and the implications for emissions and allowance prices in the EU, experts expect that about half of this quantity will be used up by 2012, even though there is no consensus on the exact figures. Indeed, the revised Directive guarantees the unlimited and automatic banking of EU allowances (EUAs), while holding CDM and JI credits for use in the EU ETS beyond 2012 involves more uncertainties. Amongst others, three factors influence the amount of credits to be used up to 2012:

- The recognition policy of CDM credits in other trading systems, such as in the US system.

- The market sentiment regarding the European Commission's mandate, under the revised Directive, to restrict certain CDM categories for the post-2012 EU ETS.

- Developments in the international discussion on sectoral crediting.

This paper assumes that only half of the 2.8 to $3.1 \mathrm{GtCO}_{2}$ credits would be available for the period 20132020. This estimate should be taken as indicative, as it depends to a large extent on the supply of CDM/JI

\footnotetext{
16 The climate and energy package was proposed by the European Commission in January 2008 (COM(2008) 30 final). The text adopted by the European Parliament in December 2008 is available at http://www.europarl.europa.eu/sides/getDoc.do?type=TA\&reference=20081217\&secondRef=TOC\&language=EN
} 
credits over 2008-12, and is based on an EU mitigation goal of $20 \%$ by $2020 .{ }^{17}$ In this scenario, the annual average demand for credits between 2013 and 2020 would be about $185 \mathrm{MtCO}_{2}$.

There is no decision on the use of credits in US climate policy. Yet, if current domestic discussions are any indication, the USA could be a major buyer on the post-2012 carbon market. The recent Waxman-Markey draft legislative bill ${ }^{18}$ calls for ambitious reduction goals ${ }^{19}$ and a cap-and-trade programme covering $85 \%$ of US GHG emissions. It proposes extensive use of reductions achieved outside the system, either internationally or domestically. A maximum of about $2 \mathrm{GtCO}_{2}$ per year could enter the system, evenly divided between domestic and international offsets, including forestry. No offset standards are specified, and the US EPA would determine eligible offset types. In order to minimise potential leakage and encourage nationally appropriate mitigation actions the bill requires the US EPA to identify sectors in specific countries where issuance of credits on a sectoral basis is appropriate. Once such a sector/country is identified credits can only be issued on a sectoral basis in that sector/country. ${ }^{20}$ The issuance of credits can only take place in a country that is party to a bilateral or multilateral agreement to which the US is also a party. The bill also states that the baseline should be set lower than a business-as-usual scenario. Also, offsets are discounted at $80 \%$ of an allowance. Experts estimate that some $800 \mathrm{MtCO}_{2}$ would be imported annually between 2012-20 (New Carbon Finance, 2009b). We therefore assume that demand for international credits corresponds to $800 \mathrm{MtCO}_{2}$, staying thus below the legal maximum of $1 \mathrm{GtCO}_{2}$.

Other regions are expected to add to the demand side, although there is no indication to which extent they intend to use credits after 2012. For example, while draft legislation for a domestic cap-and-trade system in Australia also includes no limits on the use of eligible international credits, there are also calls for restrictions. Similarly, signals in Japan indicate a more cautious approach regarding the use of offset mechanisms. ${ }^{21}$

As a consequence of considerable uncertainties, we restrict our calculations for demand to the EU and US, whose policy positions seem to be at a more mature stage, albeit far from final. With these qualifications in mind, we estimate that the average annual demand from the US and the EU for international credits could amount to roughly $1 \mathrm{GtCO}_{2}$. Other industrialised countries are likely to augment this demand, although by how much is unknown.

The supply of credits depends strongly on participating countries and sectors and on the ambition of sectoral baselines/targets, and on the effectiveness with which host countries would implement the mechanism and reduce the GHG emissions below the target. In some cases, supply could surpass the estimated demand by a large margin, especially if forestry were eligible.

\footnotetext{
17 With an international agreement, the EU would adopt a $30 \%$ mitigation goal, which would probably be accompanied by an increase in the allowed quantity of credits for compliance.

18 On March 31, 2009, the House Energy and Commerce Committee Chairman Henry Waxman and Global Warming Subcommittee Chairman Ed Markey released a comprehensive draft of a bill covering climate change and energy legislation, the American Clean Energy and Security Act of 2009 (ACESA). The bill builds upon an influential proposal by the US Climate Action Partnership (USCAP), a partnership of big business and non governmental organizations committed to urgently enacting climate legislation in 2009. To become law, the draft bill must go through the Committee at the House and obtain approval by the Senate.

19 Targets are set at 3\% reduction from 2005 by 2012, 20\% by 2020, 42\% by 2030; and 83\% by 2050. To compare, US GHG emissions were 16\% above the 1990 level in 2005.

20 The general criteria for appropriate sectors/countries are countries with high GHG emissions, or comparatively higher level of economic development.

21 Japan indicated that “the use of flexible mechanism should be allowed as a supplementary Measure” (Japan’s proposal for AWG-LCA: for preparation of Chair's document for COP 14).
} 


\section{Box 2. The role of international credits in EU climate policy}

In December 2008, the European Council and the European Parliament endorsed an agreement on the climate change and energy package, which translates into details a political commitment by the European Union to reduce its GHG emissions by $20 \%$ by $2020 .^{22}$ International credits figure prominently in helping member states reach this target. In the EU ETS, the use of CDM and JI credits is already common practice, with some restrictions on the quantity that can be imported. Within the allocation process for phase 2 of the EU ETS, credits corresponding to $13.5 \%$ of the phase 2 cap were allowed. The package extends the access rules to credits to phase 3 of the EU ETS. The EU ETS aggregate 2008 to 2020 import limit is approximately $6.5 \%$ of the aggregate cap. In addition, the agreement covers the quantity of credits that can be used by non-EU ETS sectors.

Overall, credits are allowed to the following extent ${ }^{23}$ :

- Sectors under the EU ETS can use credits worth between 1.6 and $1.9 \mathrm{GtCO}_{2}$ eq between 2008 and 2020. The total amount of credits for existing ETS sectors ranges between 1.5 and 1.7 billion tonnes $\mathrm{CO}_{2}$ eq. In addition, allocations between 0.1 and 0.2 billion tonnes $\mathrm{CO}_{2}$ eq will be available for new sectors as well as for aviation. Final details will be agreed under the EU comitology process. The principle behind these numbers was to ensure that a maximum of $50 \%$ of the EU-wide reductions of existing ETS sectors over 2008-2020, and new sectors (and aviation) over 2013-2020, could be met via the use of CDM/JI credits.

- Outside of the EU ETS, credits corresponding to about 1.1 and $1.3 \mathrm{GtCO}_{2}$ eq can be used between 20082020.

- Average annual amount of credits allowed in the EU between $2008-2020$ is thus ca. $218-237 \mathrm{MtCO}_{2} \mathrm{eq}$. This number may be increased if the EU were to agree to a $-30 \%$ target for 2020 .

In comparison, the EU ETS market is based on an average annual cap of 2,083 $\mathrm{MtCO}_{2}$ eq over 2008-2012, gradually declining towards $1,720 \mathrm{MtCO}_{2} \mathrm{eq}$ in 2020.

Figure 2 attempts to summarise the above numbers. The studies cited on power generation indicate that as much as two-thirds of possible demand could be met by the power sector alone - assuming that all major emerging economies participate. The total mitigation potential of the cement sector in Brazil, China and Mexico could meet the remainder, although no attempts at baselines were discussed here. This picture would have to be completed with the expected contribution of the CDM, and sectoral crediting estimates for other sectors. Last but not least, whether and how the forestry sector (i.e. REDD credits) would be allowed to participate in the carbon market is of paramount importance in this discussion as its mitigation potential is as large as our estimates for US and EU demands together.

Nonetheless, this summary of existing estimates and analysis of current policy proposals suggests that the issue of oversupply needs to be kept in close consideration when considering a sectoral crediting mechanism. Five uncertainties influence the global supply-demand balance, and are likely to affect these findings:

- The structural supply/demand framework arising from a possible agreement in Copenhagen, which could encourage developed countries to rely more on international credits, but could also mean ambitious mitigation actions in developing countries, not necessarily supported by the carbon market, or ambitious baselines that would reduce the crediting potential.

- The question of whether governments decide to buy AAUs and use them in the national/regional systems - as opposed to restricting their use to governments. The level of potential AAU

22 A 30\% reduction target is proposed if other Parties were to take equally ambitious mitigation objectives.

${ }^{23}$ These numbers are based on personal communications with Emmanuel Fages, orbeo, and a study by Deutsche Bank (Lewis and Curien, 2008). 
purchases both influences the demand for and supply of international credits, depending on the price discount that sellers may propose. ${ }^{24}$

- $\quad$ Price uncertainties of international credits. Demand for international credits is highly sensitive to price expectations. If international credits used for compliance in domestic/regional trading systems become more expensive than domestic units, demand shrinks. At the same time, lowerthan-expected credit prices can also lower the generation of credits, impacting thus supply and in turn credit prices. The current economic downturn and resulting lower demand for and supply of international credits demonstrates this aspect.

- Energy and other price uncertainties. The relative price of carbon-intensive energy sources impacts the fuel choice and thus emissions of actors. As a consequence, energy price uncertainties influence demand for and supply of credits. There may be a similar effect from the price of agricultural commodities, as agriculture sometimes competes with forestry for the use of land.

- Eligibility of countries and sectors. Finally, the supply of and demand for international credits strongly depend on the countries and sectors that are deemed eligible for any sectoral crediting or trading mechanism - including which entities inside a sector can participate (e.g. the EU ETS set some thresholds for participation, which may be of great importance in sectors like cement where small and large entities co-exist in some countries). Different domestic/regional trading systems may also have restrictions on which sectors are eligible to export credits into their markets.

24 To date, there have been confirmed AAU sales of about $140 \mathrm{MtCO}_{2}$. (Lewis and Curien, 2009). Ongoing discussions relate to how surplus AAU banking from the 2008-12 period could be aligned with discussions on goals, in order to avoid detrimental impacts on the carbon market and real emission reductions. 


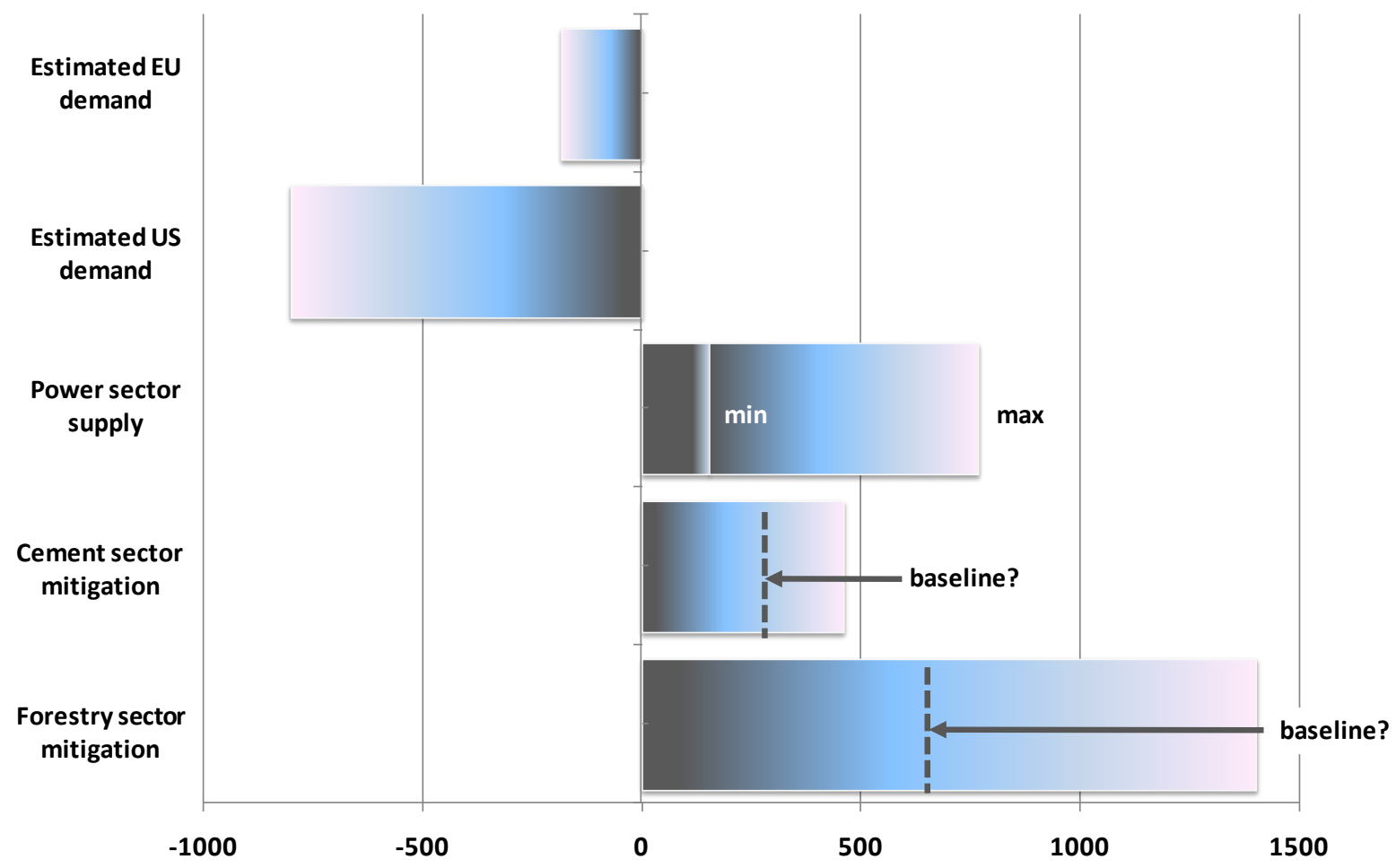

Source: Authors - see above text for full references.

Notes: The data in this graph are drawn from studies with different assumptions, looking at selected key sectors and countries.

- Only the bars for the power sector represent potential credit supply (i.e. not total mitigation potential), mainly from China and other emerging economies.

- The bars for the cement sector represent the possible mitigation potential in China, Mexico and Brazil.

- The bars for the forestry sector represent the possible mitigation potential in developing countries.

- Estimated EU demand is based on the assumption that half of the overall amount of credits allowed by the energy and climate package will be available for the period 2013-20.

- Estimated US demand is based on the assumption that the entire amount of international credits currently proposed by the Waxman-Markey draft bill is allowed.

In the design of sector-based carbon markets, further work is needed to align the ambition of sectoral baselines with realistic projections for levels of demand, taking into account countries' preferences for domestic versus international actions. A key policy challenge is the uncertainty in supply estimates due to unknown national and international future developments. Whatever crediting mechanism may be agreed, it is essential that it raises reasonable expectations about its contribution to the financing of mitigation activities in developing countries - and then about how much of the mitigation should come as a net contribution to lower global emissions (see section 4 for discussion on this issue), and what other support mechanisms may be adequate. 


\section{How Would Sectoral Market Mechanisms Work?}

Experience with the Kyoto Protocol has shown that a number of steps are needed before emissions trading (including project-based mechanisms) can become operational in countries. In other words, the adoption of national or sectoral emissions baselines or targets is a necessary, yet far from sufficient, initial step towards the diffusion of a carbon price to emission sources. This consideration is at the core of the issues studied in this section, which proposes to clarify how the two main types of market-based mechanisms could be implemented in countries.

- Sectoral crediting, with credits generated after evaluation of performance against the agreed baseline - credits are issued ex post, as is the case under the CDM.

- $\quad$ Sectoral trading, whereby allocation occurs ex ante.

Another dimension is, however, of paramount importance for the effectiveness of the market mechanism: the type of emission goal used. In what follows, we consider intensity-based and fixed targets for crediting. As for sectoral trading, we assume fixed targets.

Technology diffusion goals are also studied to see if they could be the basis for access to carbon market finance.

\subsection{Implementing sectoral crediting based on intensity goals}

\subsubsection{To credit or not to credit?}

As defined earlier, sectoral crediting could operate from a baseline expressed in intensity terms $\left(\mathrm{tCO}_{2}\right.$-eq per unit of output), or from a baseline expressed in terms of a fixed quantity of emissions, i.e., $\mathrm{X}$ tonnes of $\mathrm{CO}_{2}$-eq.

For an intensity goal, the country would collect information on 1) emissions in the sector and 2) the agreed measurement of output (tonnes of cement, MWh, etc.), to assess performance in intensity terms and compare it with the baseline. Data used to set the baseline and to assess performance would need to be verified.

The measured performance of the sector, if worse than the baseline, would generate no credits. If performance were better than the baseline (a lower intensity), crediting would accrue, for a total quantity equal to the total volume times the difference between the baseline level and measured performance. Here is a simple illustration from the power generation sector:

- The baseline is set at $0.5 \mathrm{tCO}_{2}$ per megawatt hour (MWh) of electricity.

- $\quad$ Total output is 50,000,000 MWh.

- $\quad$ Recorded performance is $0.45 \mathrm{tCO}_{2} / \mathrm{MWh}$

- $\quad$ Credits amount to 50,000,000 x $(0.5-0.45)=2.5 \mathrm{MtCO}_{2}$

At first sight, this is a mere extrapolation of how a CDM project operates with an output-based baseline: CERs are issued for avoided emissions from a baseline, taking output growth into account. In terms of incentives to actual plants and sectoral stakeholders, it is in fact quite different from CDM, because the country/sector performance is the sum of performance of several entities. We develop this notion below. 


\section{Box 3. Possible overselling via "no lose" sectoral crediting}

In this paper, sectoral crediting is defined as a "no lose” target, implying no penalties, financial or other, in case of non-compliance with the target. In Accra, the Chair of the Ad Hoc Working Group on further Commitments for Annex I Parties under the Kyoto Protocol proposed the following text in his draft conclusions: "A non-Annex I Party may propose a "no lose" target for emissions from a sector. [...]There shall be no consequences for a Party that does not meet its approved target” (FCCC/KP/AWG/2008/L.12).

This assumption is possible, as long as a country (or the instrument itself) does not allow credits to be sold until overall performance at the sector level is assessed. If credits had been transferred ex ante, beyond what ex post performance levels would allow, the country would have oversold credits. If a country over-sold credits, one would assume that it would be liable, and some "consequence" would need to be borne. To prevent this and in keeping with the "no lose" nature of the target, Parties could decide to not allow transfers of credits ex ante, as this would run the risk of generating financial revenues at the expense of the environment. The following provides an illustration of overselling for an intensity-based objective.

- A country adopts an intensity target of $0.5 \mathrm{tCO}_{2} / \mathrm{MWh}$ in its power and heat generation sector. A country relying on standard coal technology for half of its generation and a combination of hydro, nuclear and renewables for the rest would emit this much per MWh.

- In the period over which the commitment applies, it generates 10 TWh (10,000,000 MWh).

- Its sectoral emission target, in this case, is simply $0.5 \times 10$ million $=5 \mathrm{MtCO}_{2}$

- Its performance happens to be $0.51 \mathrm{tCO}_{2} / \mathrm{MWh}$. As Parties agreed to the "no lose" nature of the target, the increase over the intended goal is acceptable: $(0.51-0.5) \times 10$ million $=0.1 \mathrm{MtCO}_{2}$

- However, because good performance was recorded in one segment of the industry (say at 0.38 tCO $/ \mathrm{MWh}$, for $1 \mathrm{TWh}$ ), the government had allowed a forward transaction of the corresponding reductions: $(0.5-0.38) \times 1$ million $=0.12 \mathrm{MtCO}_{2}$.

This amount allows for a corresponding increase in emissions on the buyer's side, over and above the agreed "no lose" target, in effect undermining the integrity of the "no lose" target.

Three broad scenarios can be envisioned, with respect to individual source level performance:

1. Each individual source reduces emissions per unit of output below the sectoral intensity target adopted at the national level.

2. Certain individual sources reduce their intensity below the target, while others do not. In aggregate however, the average sectoral performance is better than the target. The above illustration could fit under either this or the previous scenario.

3. A limited number of plants act, while the bulk of the sector does not, and overall performance is worse than the baseline (i.e., a higher intensity). Here is an illustration:

0 The baseline is set at $0.5 \mathrm{tCO}_{2} / \mathrm{MWh}$.

o Total output is 50,000,000 MWh.

o Recorded performance is $0.51 \mathrm{tCO}_{2} / \mathrm{MWh}$.

- Half of the output emits $0.48 \mathrm{tCO}_{2} / \mathrm{MWh}$ on average.

- The other half emits $0.54 \mathrm{tCO}_{2} / \mathrm{MWh}$ on average.

o Credits amount to $50,000,000 \times(0.5-0.51)=-500,000 \mathrm{tCO}_{2}$. No credits should be issued to the country in this situation.

This last scenario could lead to a large reduction in the carbon price signal for participating entities, when compared to the current situation under the CDM. In the CDM, compliance is assessed at the individual project level: credits are directly linked to the project performance. Under a SCM, an individual source A 
may outperform the target, but if on average the sector as a whole does not outperform the target, then the sector as a whole and thus source A would also not be eligible for credits. In all, the structure of incentives is clearly different under sector-wide crediting. A financier asked to pay for efficiency improvements on the basis of expected carbon revenues of a plant would be worried that such revenues would depend not just on the performance of that one plant, but on the performance of all other plants in the country - and on how the government has agreed to distribute revenues among participants. Thus, the carbon price signal from sectoral crediting mechanisms may be dampened, unless accompanying measures have been taken at the domestic level.

\subsubsection{Domestic implementation scenarios and link to international market}

Without additional domestic measures, intensity-based sectoral crediting faces the problem of possible non-cooperation among covered sources and the lack of compliance/sanction mechanisms in case of nonperformance of some entities, introducing a risk of default on the credits if the good performance of some were offset by others. The solutions to this problem are not straightforward.

This problem depends first on how a government would implement a sectoral objective of a "no lose" nature. It is assumed that the country/sector is motivated to raise revenues from the carbon market. In other words, it should aim to out-perform the "no lose" target (the baseline for crediting). This is illustrated in Figure 3. So-called nationally appropriate mitigation actions (Bali Action Plan Art. 1.b.ii) could be the main instrument to improve the sector's performance from the reference case to the sector "no lose" target. Dedicated support could be granted for that purpose. These issues are discussed in Ellis and Moarif (2009) and Kim et al.(2009).

Figure 3: Crediting under a "no lose" sectoral target

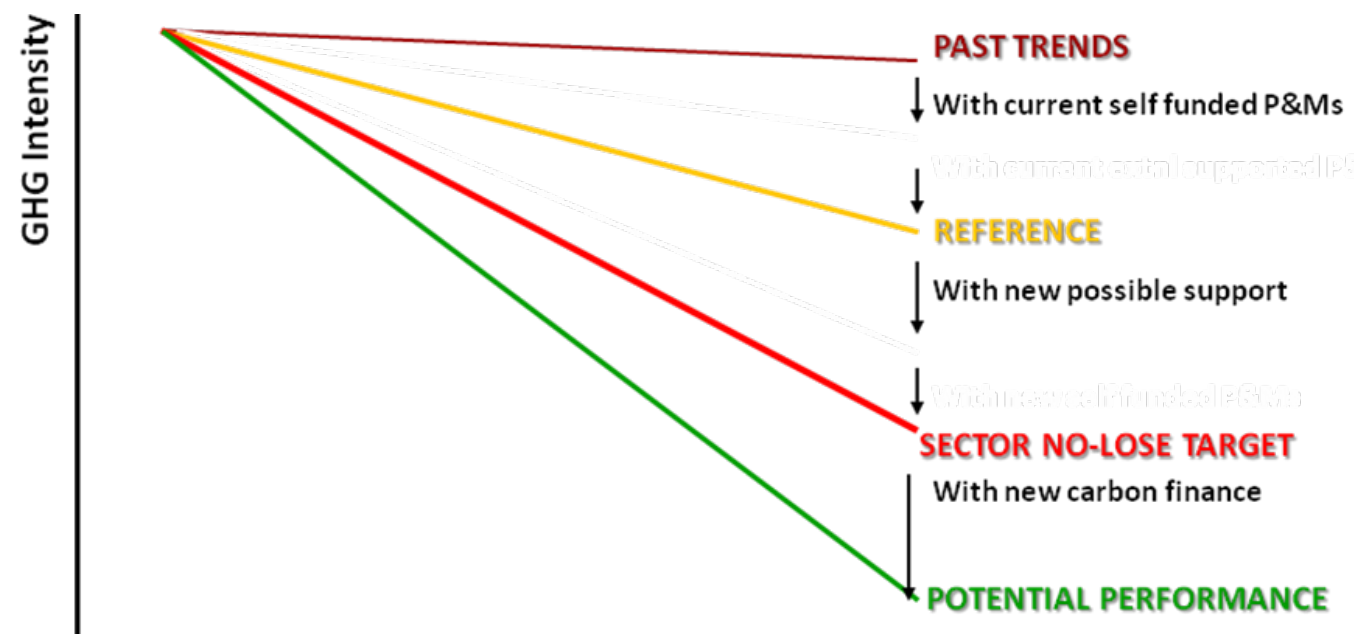

Time

Source: Ward, 2008

Various policy scenarios can be envisioned for the domestic implementation of a "no lose" sectoral target or indeed any other form of sectoral crediting. We provide a list of options, each responding to possible priorities and concerns at domestic level. They would require varying levels of intervention from national governments. More importantly, the effectiveness of the carbon price signal would also differ: while some options stop the carbon price signal at the government level, others seek to channel it as far as possible to domestic entities. Our examples are primarily drawn from power or industry, but may equally apply to other activities where an intensity goal would be feasible. 
- Revenues to government - A government may introduce a domestic policy to bring the sectoral performance in line with a goal that goes beyond the "no lose" target. Policy options for that purpose may include: mandatory energy (or $\mathrm{CO}_{2}$ ) efficiency performance standards; a share of renewable energy in power generation; the closure of small and/or highly polluting plants. The policy, if effective would allow the sector to beat the baseline. The government would however keep all credits generated for the general budget; it may also decide to allocate some of the revenues from sales of credits to firms, or finance further improvements in this or other sectors. For example, a government may use the carbon revenues to (partly) finance subsidies for renewable power generation. In this way, the private sector would not directly receive the carbon price signal but the government would provide pass the carbon price incentive on to the private sector through other mechanisms. The actual carbon price signal would not, however, be received directly by investors in the sector; on the other hand, the new policy could be of a binding nature and require private sector and other entities to comply. There is always the risk, in such circumstances, that entities are asked to incur either too high or too low a cost compared to the prevailing carbon price. ${ }^{25}$ The Appendix provides a numerical example based on a simple situation in the power generation sector, with two groups of entities, some performing better than the baseline (A), while the other is performing worse. On average, the country outperforms the baseline, but does not generate enough credits to fully reward Group A. We rely on the same basic illustration for all other scenarios, with alterations when needed.

- Credits to sources in compliance - With the same domestic policy tools, the government could decide to reward mitigation among the various entities by distributing credits based on their individual performance. But no entity could be assured at the outset that all its reductions would be matched with an equivalent quantity of credits - and the revenues that they bring. This uncertainty would blur the signal to the carbon financiers.

- Performance reserve - The government may introduce a domestic policy goal that mandates performance improvements going beyond the goal established in the other proposals, and commit to reward overachievement of the goal with credits. In case some entities were to fail to meet the more stringent domestic goal, overall performance may still be enough to reward others for their actual reductions below the baseline agreed internationally, and credits could be distributed accordingly. This measure would be equivalent to setting aside a reserve of credits, equal to the difference between the agreed international goal and the more ambitious domestic goal. The reserve would be a buffer against the possible non-compliance by some domestic entities.

- Government liability - The government decides to go beyond the "no lose" nature of the objective and commit to restore the environmental objective in case of over-selling (see Box 3). Under this scenario, the government would issue credits to domestic entities that perform well, with the possibility to trade these on the international market. If it then turns out that these sales, when counted against the country's performance, imply that it sold more than its overall achievement allowed, the government would commit to make up for the difference by acquiring valid units from the international market. ${ }^{26}$ This may allow for a more straightforward signal from the carbon market to investors, but it also means that a government would face some financial burden if overselling were to occur. Further, this may lead to unnecessary price fluctuations if there were large-scale overselling during the period as a result of over-allocation,

25 This is a common criticism addressed to regulatory, 'command-and-control', measures as a solution to an environmental externality: mandating a technology response may impose a cost that is out of proportion with the recognised social cost of the externality. Cap-and-trade and taxes are therefore preferred, in an ideal market setting.

26 We note that under the Kyoto Protocol, no Party is obliged to restore its assigned amount to a level that matches emissions: it can try to make up for the generated 'debt' in the following period, with a $30 \%$ penalty. 
followed later on by a massive acquisition by governments to "make good" on the environmental damage created by overselling.

This option could mean that credits were issued before the international assessment of the target has been conducted. Alternatively, the government may be involved in forward transactions for such credits, with the commitment to provide the needed credits from the international market if the international credits couldn't come in numbers high enough to reward all good performers. Another way to facilitate this would be that performance be assessed every year and credits be issued on that basis - if so, bad performance one year would not affect credits issued the previous year or the next; ${ }^{27}$ this option may lower investors' uncertainty, but more frequent reports and verification would add to the administrative cost of the system.

Figure 4: Sectoral crediting option - Credits to sources in compliance

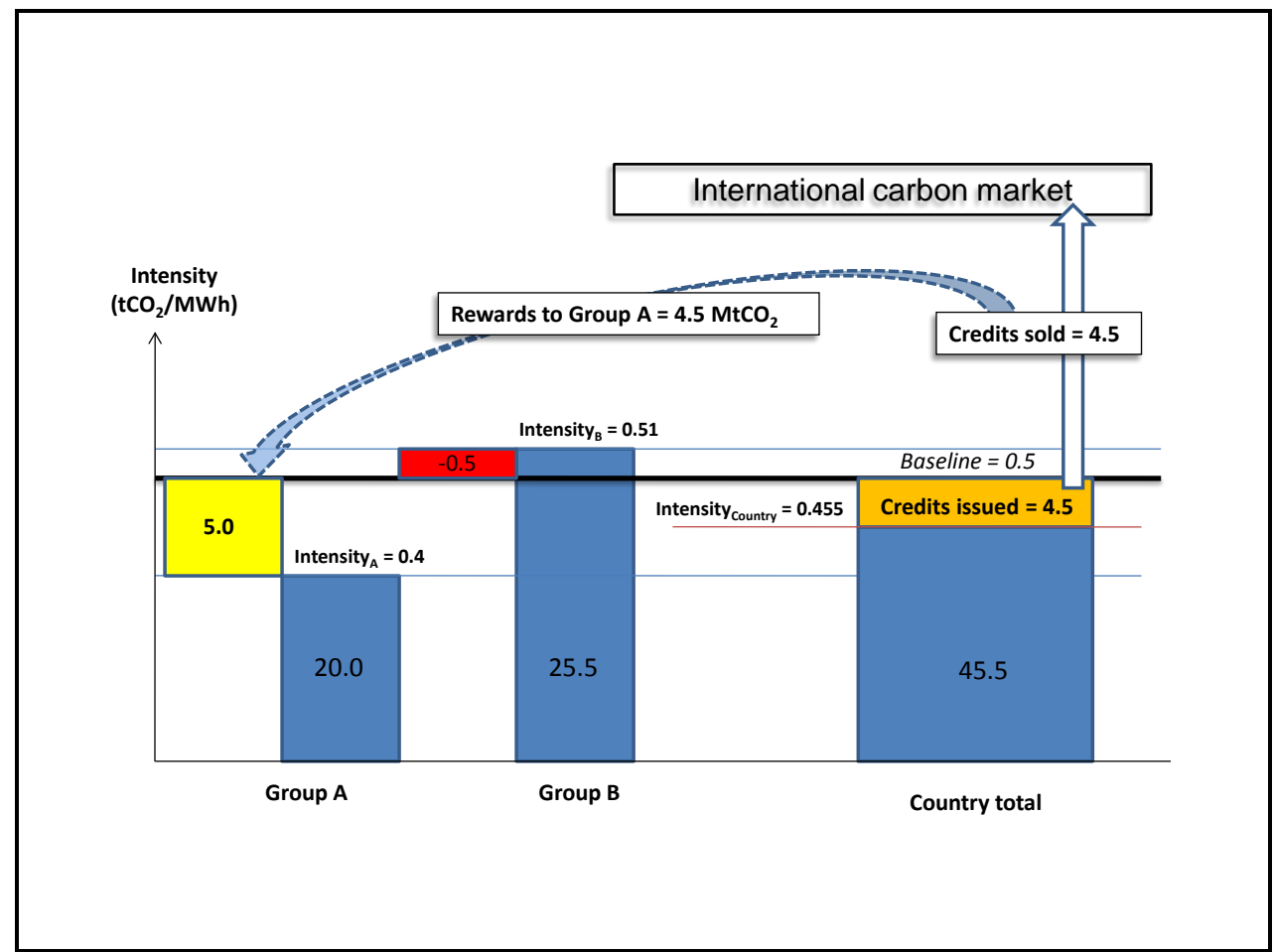

Note: Under this scenario, Group A contributes all of the country's reductions from baseline - Group B deteriorates overall performance. Group A cannot therefore be rewarded for the entirety of its reductions. As only $4.5 \mathrm{MtCO}_{2}$ get sold on the international market, Group A could expect this as maximum revenues. Any further deterioration in Group B would lower that amount.

27 This is defined as a gross approach to crediting; a net approach would look at performance over the whole period and credit for the average performance. In essence, this is about turning a "no lose" target that spans several years into a series of annual no-lose targets. 
Figure 5: Securing credits with a performance reserve

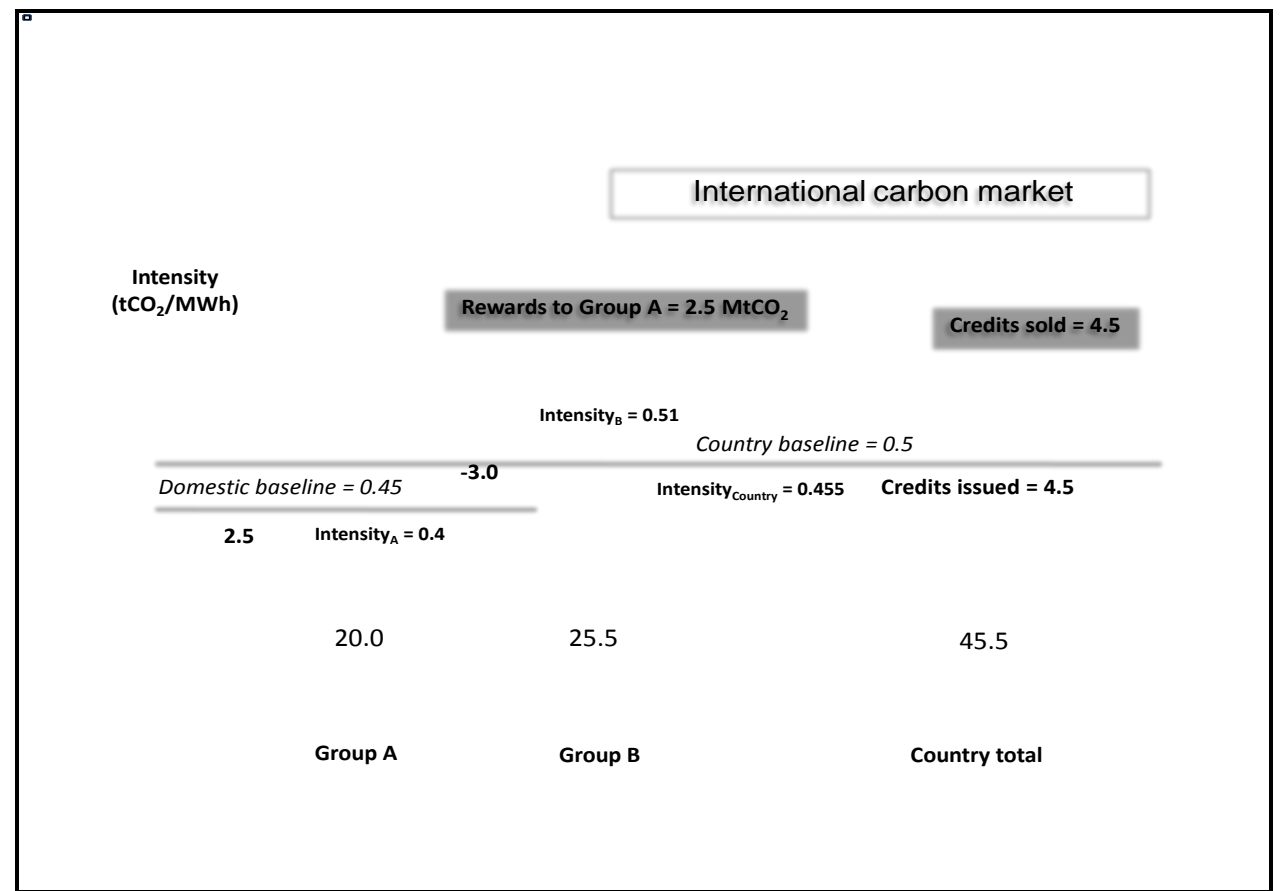

Note: A domestic baseline is introduced, at a lower level than the internationally agreed one, to increase the certainty that credits generated by some entities will not be cancelled by other less performing ones. This of course implies lower overall revenues for investors, but higher certainty on the credit amount. The government may also decide to reward good performance with the excess credits generated at the country level.

Figure 6: Government assumes liability for any overselling

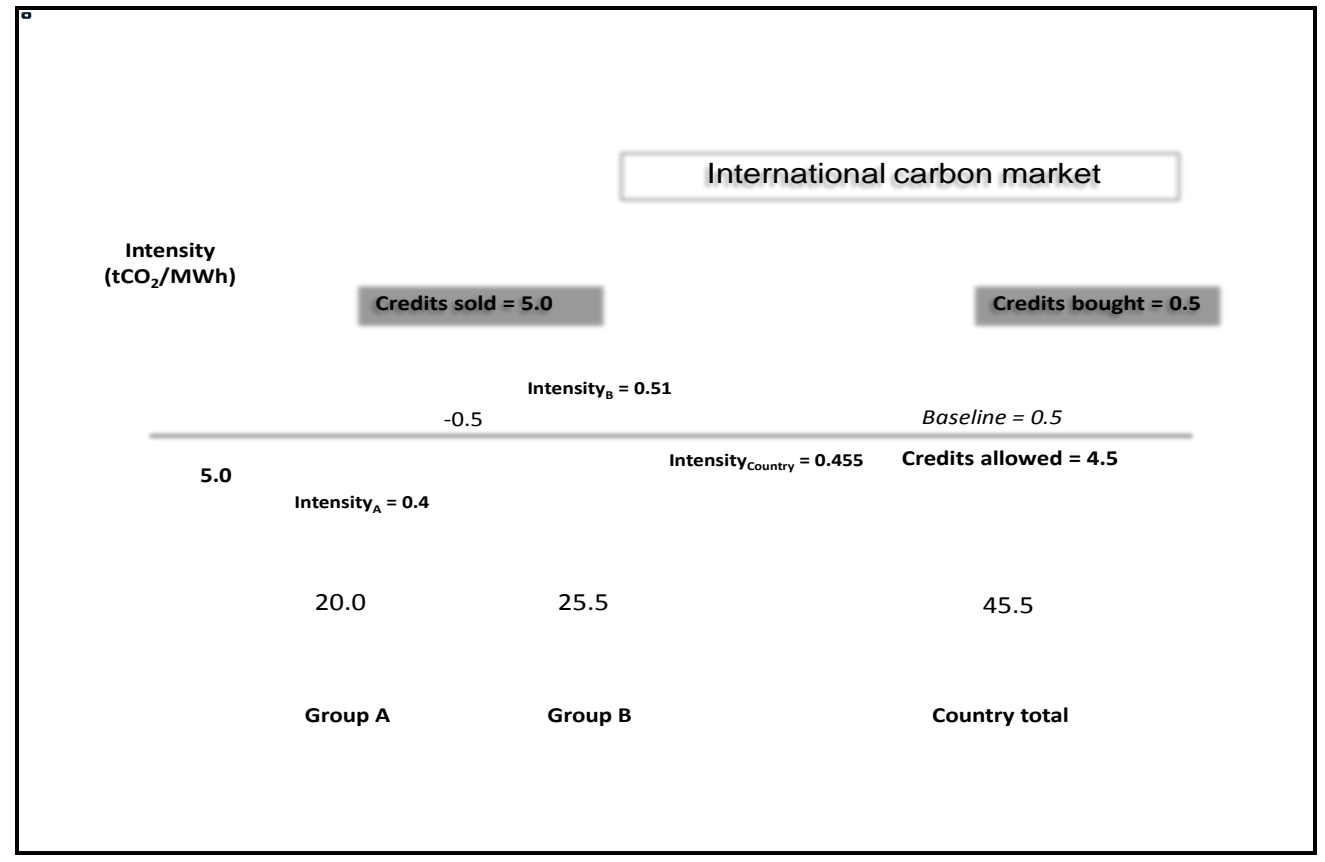

Note: The government allows good performance to be rewarded on the carbon market but assumes full responsibility for any overselling at country level. In this example, it would buy back 500,000 $\mathrm{tCO}_{2}$ from the market to restore compliance with its crediting baseline. It may also decide to fine Group B for that purpose. 


\subsubsection{Summary}

The sections above describe how governments could organise the link between an international sectoral crediting objective - and the possible generation of credits - and efforts by domestic entities, ranging from a separation from the price signal (credit revenues go to the government) to an attempt at a full link (with full liability assumed by the government in case of overselling). This list is by no means complete, nor does it provide all details for implementation of the objectives at domestic level, but it gives an idea of how far governments could go to secure a carbon price signal for investors in the country, once it had adopted a ("no lose") intensity-based sectoral target. It appears that the effectiveness of the price signal requires that a more stringent system be put in place, to ensure that all entities are encouraged to improve performance. This is essential to avoid the problem of diffuse incentives to individual players presented at the end of section 3.1.1. There may of course be cases where entities are fully regulated, or do not operate in a competitive environment, in which cases getting the carbon price signal to plants/entities matters less: it would be up to the regulatory environment to reflect the new policy direction, needed to achieve and outperform the "no lose" goal.

Implementation of intensity-based goals in cases were individual plants or companies make their own investment decisions would not be trivial. As entities' performance are likely to differ, decisions will need to be made on which performance, or improvement from past performance, is allowed to generate credits, and which is not. In our illustrations, we assume two homogenous groups of entities (good performers, and bad performers); the reality is likely to be more complex. For instance, a country that agrees to move toward low $\mathrm{CO}_{2}$ emissions in power generation could agree to credit ultra-super critical coal plants, as a marked improvement from its existing capacity. Alternatively, it could only allow credits to go to generation technologies with much lower emission levels. What these choices would imply in terms of incentives and overall environmental effectiveness of this intensity-based market mechanism is not clear.

In all, sectoral crediting represents a significant policy effort on the part of the host government, if it is to generate credits and fully benefit from the carbon market revenues. For some of the implementation options described above, the government would also have more liability compared to the CDM; the "no lose” objective would not be without consequences if the goals were not met.

\subsection{Fixed sectoral targets}

Sectoral market mechanisms could also be designed based on fixed (absolute) sectoral targets, i.e. in terms of $\mathrm{t} \mathrm{CO}_{2}$-eq. emissions for a particular sector in a given time period. The term 'allowance' is used here as the unit for transfer under a sectoral emissions trading scheme, where allocation to the country/sector is done ex ante. This is in opposition to 'credit' refers to an emission reductions from a previously-agreed baseline (developed either on a fixed or intensity-based manner), only issued ex post after verification of performance against the baseline.

The current debate on sectoral market instruments for developing countries suggests two options:

- Sectoral crediting, which if developed on a fixed basis could mean a 'no lose' fixed baseline, whereby a country could sell credits if emissions are lower than the fixed baseline, but would not be obliged to buy if emissions were higher.

- Sectoral trading, whereby a sectoral cap is agreed, allowances can be bought and sold, and the country is internationally liable in case of non-compliance.

These approaches to developing fixed sectoral targets or baselines could be similar to each other in some respects (e.g. data needed to develop the baseline or target level), but differ in others (e.g. allocation, the implications for governments in terms of revenues and liability). 


\subsubsection{Pros and cons of fixed and intensity targets}

There are both advantages and disadvantages of developing fixed targets/baselines, as compared to intensity ones.

The main advantage of developing a fixed baseline is the certainty on the maximum level of allowable emissions from a given sector. This certainty is accompanied with an easier monitoring process as a single measure is needed to assess compliance - sectoral emissions, as opposed to emissions and an indicator of output in the case of intensity objectives. Fixed targets for GHG emissions may also be more appropriate for a limited number of sources/sectors , such as HCFC22 production where an intensity-based target could provide perverse incentives to increase production of HCFC22 in order to generate a corresponding increase in credits for reducing emissions of the by-product HFC23.

One often-mentioned disadvantage of fixed baselines is their lack of flexibility with respect to the growth of the sector, and what this implies for entities. For instance, unexpected growth in activity is likely to make it harder to meet a fixed emissions target, whereas unexpected growth combined with an intensity target, could lead to more credits. Conversely, structural changes could make fixed emissions targets easier to meet than initially expected (although this may be more relevant for national, rather than sectoral, intensity targets) whereas in an economic downturn, a sectoral intensity goal may be more difficult to reach in some cases. This would be the case if lower investment lead to fewer opportunities to introduce productivity improvements and lower emissions. ${ }^{28}$ In the definition of fixed targets, the risk of higher-thanexpected output growth could lead to an inflation of the target during the negotiation. This has clearly been the case in the first phase of the EU ETS, where governments had limited data to evaluate industry projections on future output and related emissions (Ellerman and Buchner, 2008). Developing intensity targets in a rigorous manner would require more precise information on production methods, possible improvements and costs; however, a country that envisions an absolute sectoral goal would also need some information in intensity (although maybe less). In all, if a hard cap is less data-intensive on the monitoring, reporting and verification side, it nonetheless needs output projections - very often uncertain - in the capsetting phase, information that would be less critical intensity-based baselines.

Another possible advantage of an absolute target is the potential ease of devolution to actors responsible for emissions, even if distribution of cost across entities will always be sensitive. Options to allocate the emission goals are well known, from grand-fathered allocation based on past emission levels, a gratis allocation based on a sectoral benchmark, or an auction, with the option to recycle revenues to offset possible negative impacts (NERA, 2002). Allowances, in the hands of an entity liable for its emissions, ought to be a better incentive for mitigation than credits, the quantity of which is uncertain until countrywide sectoral performance has been assessed.

Environmental effectiveness is obviously also a key issue. How this is affected by a sectoral trading or crediting mechanism, however, depends on the level of ambition/stringency of the target or baseline used, rather than on how this target or baseline is established. Thus, without knowing the baseline level, it is not possible to indicate whether a crediting mechanism based on a fixed baseline could be more or less stringent/generate more or fewer credits than an intensity-based baseline.

Irrespective of whether targets/baselines are intensity-based or fixed, governments face similar issues and need to make domestic policy choices on the following questions:

- Allocation rules within the sector, i.e. how to share the effort among sources, with the inherent complexity coming from the diversity of performance and installation-specific circumstances. 28 There could be cases where the opposite occurs: a lower demand for electricity could reduce peak load production
and, with it, the use of more $\mathrm{CO}_{2}$-intensive fuel turbines. 
- Whether governments wish to benefit from any revenues generated by a sectoral crediting or trading mechanism (and if so, what proportion of any revenues would go to them as opposed to entities).

- How to best incentivise individual entities to improve their performance such that the whole sector's emissions are below the target or baseline. The incentive is likely to be clearer for allowances derived from a fixed target. Crediting, as an ex post mechanism, faces the problem highlighted in section 3.1.1: the carbon price incentive may not easily reach the covered entities, whose situations also differ in terms of GHG performance and in cost-effective GHG mitigation potentials. The government may also decide to apply a regulatory measure to trigger changes and not directly expose entities to the carbon price.

- Liability: whether the government would decide to reward good performers even if the sector as a whole emits over its baseline, and if so, who would be liable for "making good" such a reward (e.g. the government, or poor performers in the sector).

\subsubsection{Sectoral crediting mechanisms with "no lose" fixed baselines}

A baseline expressed as a fixed quantity of emissions (rather than in intensity terms) could also be the basis of a "no lose" crediting mechanism: a sector in a developing country could generate ex post credits if its emissions were below the agreed fixed baseline. No penalty would apply otherwise.

If such a mechanism were to be implemented, government decisions on how to allocate emissions credits would be crucial in determining the level of possible benefits for participating entities. Thus, having a fixed baseline raises issues in terms of how to balance encouraging good performance, with who should benefit from the "no lose" aspect of the mechanism if the performance of the sector does not meet expectations.

This is illustrated in the following example and figures 7 and 8 . In both cases, current emissions of the sector are 50 units, and the baseline for the "no lose" sectoral crediting mechanism is set at 45 units. For simplicity, we assume that there are only two groups producing output in this sector - Group A and Group $\mathrm{B}$, with the same level of output. In the first example, both groups reduce their emissions from 25 to 20 units. In such a case, total sectoral emissions will be 40 units, 5 below the baseline. The corresponding credits can therefore be shared between Group A and Group B (figure 7).

The situation becomes more complicated if the performances differ: one Group emits below its baseline and the other(s) above (Figure 8). Group A has made even more reductions than in the example above, but Group B has not reduced emissions at all. Total sectoral emissions in this case are equal to the sectoral baseline, although Group A has emitted below its entity baseline, and Group B has emitted above its entity baseline. In such a case, the government would need to decide whether to reward Group A, and if so, whether it should fine Group B in order to do so. This scenario would mean that the mechanism as a whole could remain "no lose" for the government, although not for entities within the sector.

A fixed target - even if "no lose" - is more amenable to a straight cap-and-trade system at domestic level, for which incentives to reduce emissions are clear, provided that enforcement is credible. In this case, Group A would sell to Group B or, if Group B did not rely on the market, it could face a domestic penalty that could reward Group A. This is of course a hypothetical scenario, but one that is easier to envision that with intensity-based objectives, which make domestic trading much more unwieldy. 
Figure 7: "No lose" sectoral crediting mechanism (with fixed baseline)

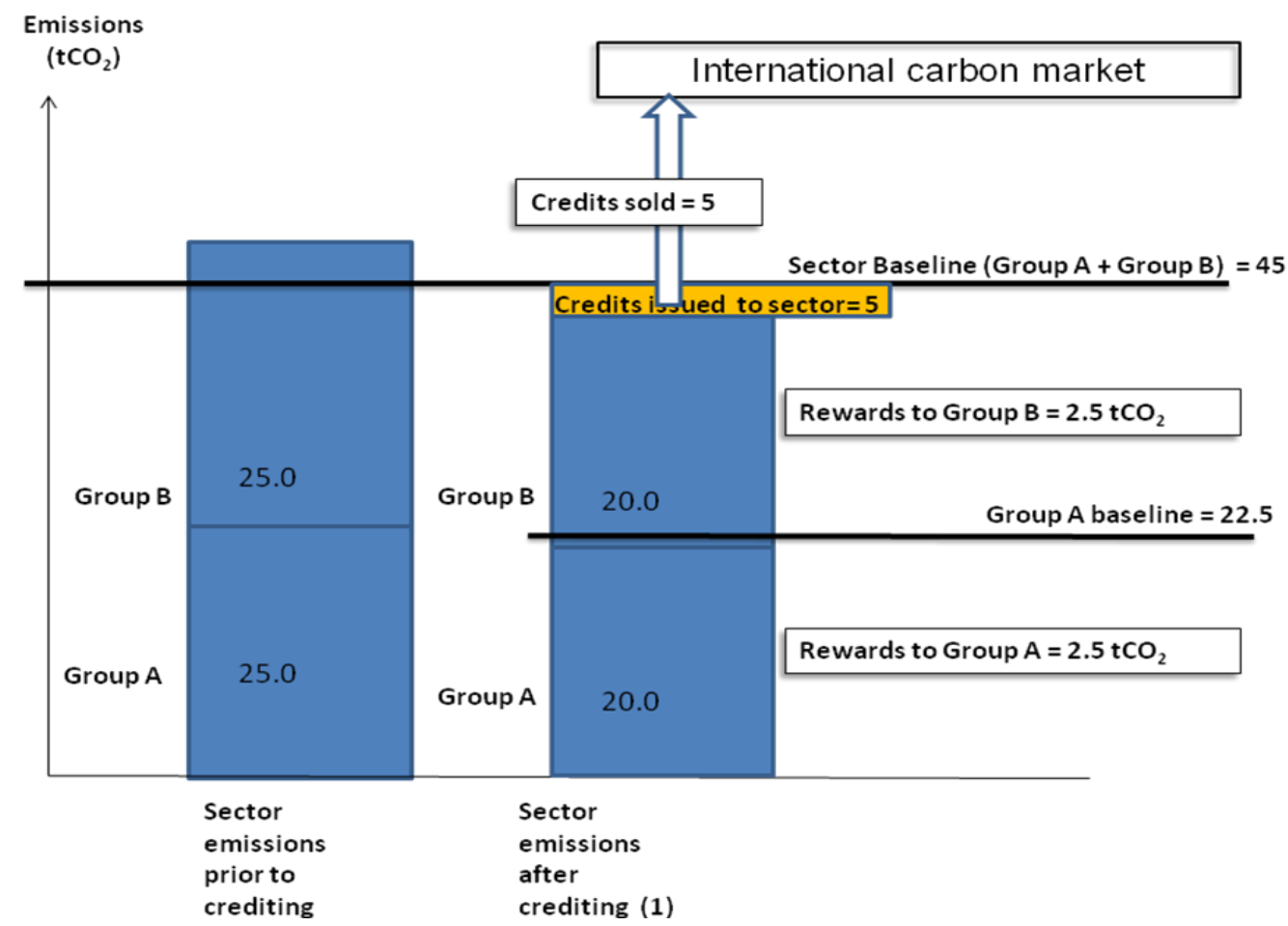

Figure 8: "No lose" sectoral crediting mechanism with a fixed baseline - balancing incentives and liability

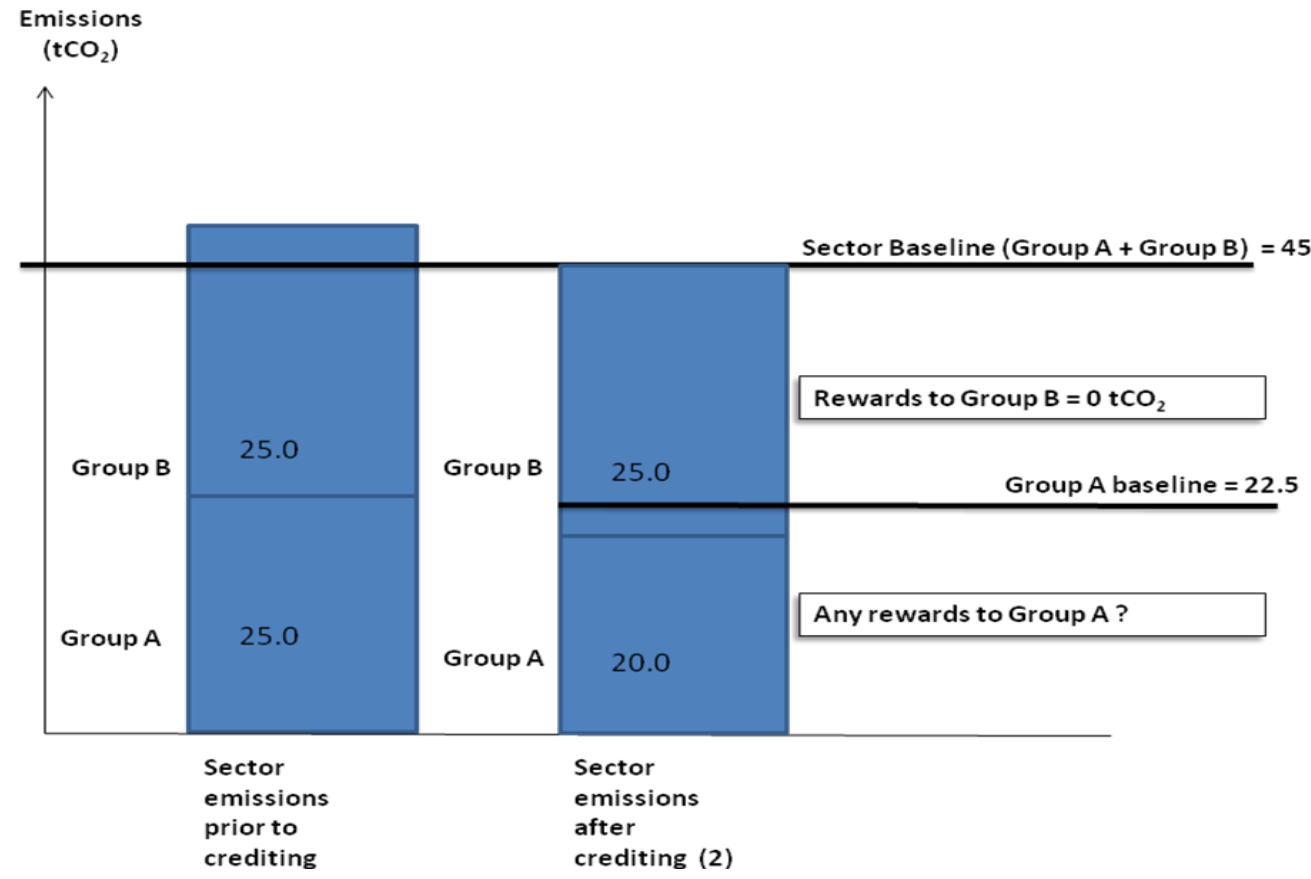




\subsubsection{Sectoral trading}

Sectoral trading presents a different type of interaction between a country's sectoral objective and the carbon markets. Under sectoral trading with a fixed target, a given sector in a given country would have an agreed emissions level in a specific time period. The allocation to the sector/country would be made ex ante, enabling a government to devolve allowances to entities in the sector and to allow them to trade nationally and internationally. As in the case of the Kyoto Protocol, there is no obligation to do so, but the government has this possibility, knowing that it assumes liability in case of non-compliance.

If sectoral approaches are to broaden the signal of the carbon market to entities that have so far been insulated from it, an ex ante allocation offers a clear advantage over ex post credits, especially when these credits are intensity-based. The allowances allocated to entities can be managed as an asset, with a clear market value, and mitigation investment could be directly rewarded by selling credits on the carbon market, without having to wait for a sector-wide assessment of emissions performance. The essential element here is of course the government assuming full liability in case of non-compliance.

Sectoral trading with fixed target levels already occurs in several developed countries, e.g. among EU27 and Norway. A key difference however is that sectoral allocations here (unlike any stand-alone sectoral emissions target in a developing country) are part of nation-wide commitments under the Kyoto Protocol, with stringent reporting and inventory obligations. As such, they did not need negotiating under the UNFCCC.

With proper reporting requirements at sector level, developing countries could engage in sectoral trading in a post-2012 climate framework. In order to allow trading by individual entities, governments would need to devolve the sectoral emissions target, i.e. develop allocation rules. Governments would also need to decide whether they keep some of the revenues that could be generated by sectoral trading in their country, or if they fully devolve the allocation to entities - governments would also have the option to "tax" trading revenues.

The assumption of a country assuming full liability in case of non-compliance does not by itself protect from the risk of overselling. However, developing a rule for sectoral trading, along the lines of the commitment period reserve used under Article 17 of the Kyoto Protocol, could help in this respect. In this case, the seller's registry, where sectoral allowances are shown, would need to keep a minimum level of allowances at all times.

\subsection{Transition issues}

Developing and implementing a sectoral crediting or trading mechanism will require institutional capacity and data - as well as political will. These can take considerable time (and resources) to develop, and not all developing countries will be willing or able to implement similar mechanisms in the same timeframe. It is therefore likely that there will be a transition period whereby some developing countries move gradually from implementing CDM projects, to undertaking GHG mitigation measures at a broader scale. There could also be differences in the type of mechanisms that various countries accept to implement (crediting, trading, with what type of emission goal).

At present, there are no sectoral-based crediting or trading schemes in developing countries. Mexico and Korea have indicated that emissions trading was one of the options considered for domestic implementation, however. Some developed countries have also indicated that they anticipate a greater level of GHG mitigation action by those developing countries which have greater responsibilities and capabilities. ${ }^{29}$ For example, reference has been made to "intensity targets" for developing countries, or for certain developing countries to join Annex I or to take on comparable commitments. Sector-specific goals are also proposed.

29 See e.g. EU, Japanese, New Zealand, US submissions to the AWG-LCA, April/May 2009. 
Without prejudging the outcome of negotiations on these sensitive topics, it is worthwhile to consider how a particular choice of policy instruments early on may be a useful step towards more ambitious actions later on or, to the contrary, make such transition difficult. This is a question that is relevant to many aspects of the negotiation that are not in the scope of this paper, but it also applies to sector-based market mechanisms. In essence, there are several different possible measures that countries could agree to before moving to a sector-wide fixed emissions target; it is therefore timely to explore which possible pathway might be the most efficient and effective. This is illustrated in Figure 9 below.

Figure 9: Possible pathways to trading with fixed caps

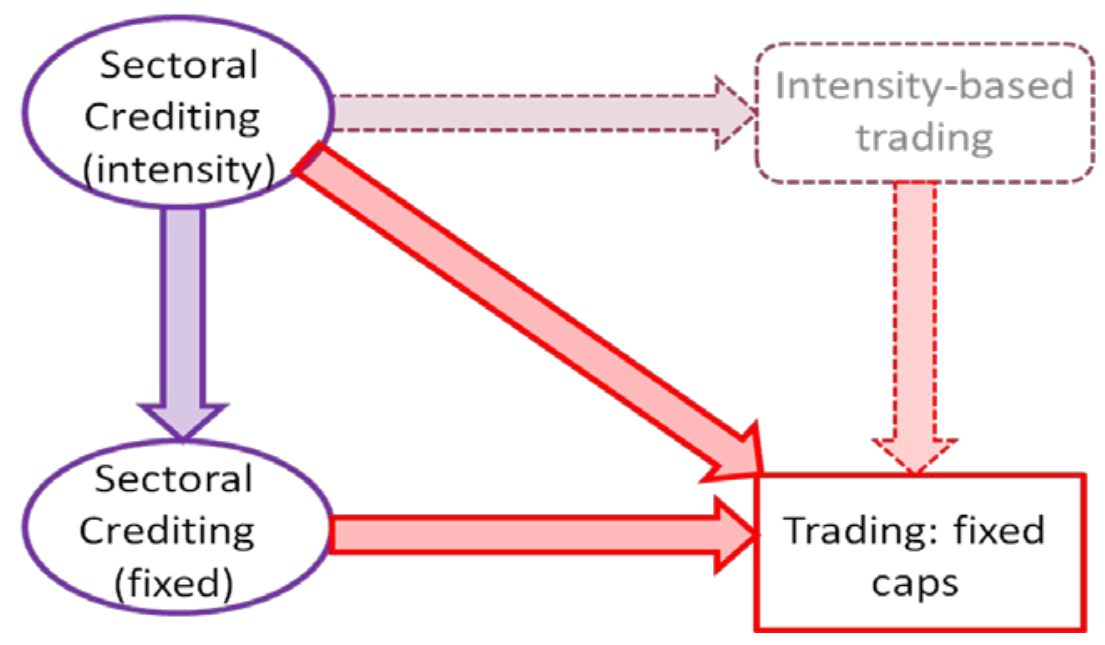

Note: the move to intensity-based trading appears unlikely, as it brings very limited advantages. The binding element of trading would not guarantee a given quantity of emissions, and the issuance of allowances when they depend on performance per unit of output would be unwieldy. A reserve to avoid overselling would also be difficult to design without any reference to a fixed target. The option is therefore not considered further in this paper.

As outlined earlier, there are data requirements and institutional implications at the national level when setting up or participating in any carbon market mechanism. This is true for mechanisms based at the project or sector level, whether or not the mechanism has been designed for credits or allowances, and irrespective of whether the mechanism has a baseline (or target) developed on a fixed, or an intensity-basis.

Nevertheless, there are some similarities between some of the mechanisms, e.g. in terms of data collection, methodology to develop baselines/targets that could facilitate a transition from one mechanism to another. For example, the task of establishing baselines for individual entities in a sector under a fixed-baseline crediting mechanism would not differ from the allocation process for sectoral trading. A sectoral crediting mechanism using fixed baselines could therefore be a sensible stepping stone to sectoral trading with fixed caps, although there could be a significant time-lag from going between one mechanism and another.

Another important transition issue is that for countries where sectors that are candidates for sectoral crediting or trading include entities that are currently participating in the CDM. The crediting lifetime for CDM projects can vary between 7-60 years depending on the type of project and whether the initial crediting period is renewed. A shorter-term transition question could therefore be how to implement a sectoral crediting (or trading) mechanism for sectors in countries who currently host CDM projects in that sector.

One key issue in the issue of transitioning from one mechanism to another is the incentives to do so. Thus, for example, there are few incentives for entities in developing countries to move from a situation where 
they can voluntarily participate in CDM projects and obtain up to $100 \%{ }^{30}$ of the revenues associated with its emission benefits, to a situation in which they may obtain only a proportion of these credits (e.g. under a "no lose" sectoral crediting mechanism), with the added uncertainty related to the country's overall performance.

Any mechanisms that are set up as a transitional mechanism towards a desired goal, e.g. sectoral trading with fixed targets, could incorporate a sunset clause. This sunset clause could be in terms of timing (e.g. countries are eligible to use this mechanism for a certain number of years), or in other terms (see e.g. Karousakis et al 2008).

An important element of this discussion relates to the domestic policy changes needed to achieve a transition from intensity-based objectives to sectoral trading. This section described earlier the many ways that a government could follow to implement a domestic intensity-based sectoral objective. This introduces uncertainty on this transition question, as starting points could vary greatly. Following is a brief illustration based on the example of the power sector.

A government may decide to implement an intensity objective $\left(\mathrm{tCO}_{2} / \mathrm{MWh}\right)$ through a penetration target for generation from renewable energy sources (RES); it could also provide subsidies to the retrofitting of existing fossil-fuel plants, or make old inefficient plants illegal and impose a mandatory minimum energy conversion standard for new plants. None of these options clearly pave the way for cap-and-trade, but neither do they necessarily make it difficult to introduce later on. By monitoring progress with these goals, the government nevertheless acquires data on performance that can only help for the elaboration of a sectoral goal. On the other hand, intensity-based crediting, and the measures described here, do nothing to address the demand-side, while it ought to be a primary contributor to lowering overall emissions - while not necessarily improving the $\mathrm{CO}_{2}$ intensity of output. Would a country decide not to act on end-use efficiency as a result? At the very least, it would not be in the power generators' direct interest to do so.

What if a country had allocated efforts to entities on the basis of intensity performance? How could it, then move towards fixed objectives, more conducive to sectoral trading? One advantage of having intensitybased performance information is that it would be easier to evaluate and compare mitigation potentials across entities, and facilitate a discussion on fixed emission goals. Again, the EU ETS has shown that such data was crucial in deriving emission caps for sectors.

There is currently no experience with such a transition, and with so many different implementation scenarios for intensity-based objectives, it is difficult at this stage to evaluate how easy or difficult it would be to move from one policy approach to the other, for the same country/sector. Ultimately, technical details matter, but most of all the heart of the matter is how one moves from a situation where the carbon market provides a subsidy for GHG reductions to one that provides flexibility to achieve emission reduction goals. It may thus be useful to have government and sectors alike well aware of the desired evolution, so that they can anticipate it.

\subsection{Getting started on sectoral emission goals}

There is now broad understanding on the data and analytical requirements to pave the way for sector-based crediting mechanisms (see in particular CCAP et al., 2008; Japan's submission on sectoral approaches, 2008; Ellis and Baron, 2006). Once political will to move forward is established, efforts should be geared towards gathering the minimum information basis to allow progress. This is of particular importance for activities where the risk of carbon lock-in is most prominent, or where the potential emissions are of a oneoff nature, such as REDD. 30 The actual level may vary depending on whether the host country government introduces a levy on CDM project
revenues. 
The above two sections touch on related questions that may need to be taken into account when considering implementing sectoral crediting. First, there is a potential for significant oversupply of credits if sectoral crediting is adopted for large emission activities and if baselines are too close to business-asusual; this would trigger a massive drop in international carbon prices. While such news would be welcome if it corresponded to technological breakthrough allowing saving $\mathrm{CO}_{2}$ at low cost, it would in this case simply reflect an inadequate choice of baseline, and correspondingly less ambitious GHG mitigation overall. Second is the issue of the effectiveness of the carbon price; the question here is: how can the carbon market be used most effectively to curb the growth of GHG emissions? To this question one would add: how can the new mechanism be made effective quickly, in light of the above-mentioned data requirements? Here are some possible elements to start answering these questions.

- Parties could rely on existing data provided by international industry groups, with measurement protocols such as the WRI-WBCSD GHG Protocol as a methodological basis to discuss baselines. Such data would need to be verified by governments before they could form the basis for a market mechanism.

- Parties could decide to dedicate additional resources to data gathering, along the lines of the IEA indicators work (IEA, 2007), to evaluate and compare performance, and then establish benchmark performance, taking local circumstances into account.

The EU ETS, and before it, the $\mathrm{NO}_{\mathrm{x}}$ and $\mathrm{SO}_{\mathrm{x}}$ programmes in the US (OECD, 1992), provide important lessons on the data issue. First, it is clear that not all sources in a sector can, nor should, be covered. Some installations are usually deemed to be too small to matter, and represent significant diseconomies of scale in terms of administrative cost for the regulator; small sources are also generally ill-equipped to participate in a cap-and-trade mechanism. Second, obtaining a proper emissions inventory for activities is of paramount importance. The main lesson is that a choice must be made on the perimeter of action. In recent work over a CDM methodology, the cement industry developed the notion of two-tiered baselines, with differentiated treatment for existing and new plants. While this was motivated by the capital stock reality of the industry and not by data, this may indicate a way forward on sectoral crediting.

One possibility would be to restrict sector-wide crediting to new plants - this is implicit in Amatayakul et al. (2008), in their estimates of credits for the power sector. This may be appropriate for sectors with very high growth, as future capacity would soon outgrow existing ones. There would, however, be no incentives for improvements in existing plants, where cost-effective mitigation potential may exist - this could be perceived as unfair. It is conceivable that CDM would continue to be used for these actors, however.

Another option is to set a relatively high capacity threshold for participation, consistent with plants that can be easily identified and monitored; the threshold for participation could be lowered over time. This would require an adjustment of the baseline as new entities would affect overall emission levels and average performance, a process that could prove cumbersome.

\subsection{Sectoral approaches and technology objectives}

Some of the options discussed for sectoral approaches combine specific support to mitigation, e.g., in the form of finance for mitigation technology. For some sectors, a technology-diffusion goal may be appropriate to reduce GHG mitigation, and could indeed be supported internationally. In this section, we envision how an approach based on technologies could be linked to GHG crediting.

\subsubsection{Defining technology-based approaches}

The possibility of crediting based on technology objectives has only recently been raised (Duan 2008, CCAP et al., 2008), so there are few details of how exactly this type of crediting may work. This paper assumes that crediting based on technology objectives would have the following characteristics: 
- Technology objectives would be specified by sector (or sub-sector). ${ }^{31}$

- Objectives could take different forms, e.g. a share of the physical capacity of a sector to be fitted with a certain technology by a specific date; increasing the capacity of technology Y by a specified amount or rate; or a share of a sector's output to be produced by a certain process.

- The agreed technology goal would form the baseline. The corresponding mitigation could either be agreed on ex ante or be assessed ex post.

- The monitoring and verification of the intended technology diffusion would have to be defined at the outset and, to the extent possible, include indicators of effective use of the technology, in order to link physical installations with actual GHG mitigation.

\section{Box 4. Technology definitions and its role in environmental policy}

There is a range of possible definitions for what a technology could constitute, when it comes to sector-level mitigation actions. For some, the GHG implications will be rather obvious (e.g. carbon capture and storage); for others less so (e.g. displaced peak electricity demand through more efficient lighting appliances); for another group, data will be needed ex post to estimate emission reductions.

Definitions of technology could vary from more narrow to much broader categories, e.g.:

- Specific hardware (hybrid vehicles; wind turbines; efficient light-bulbs)

- A process type (e.g. dry kilns for clinker manufacture, waste heat recovery, carbon capture and storage etc.)

- Broad class of technologies/energy sources (clean coal; renewable energy sources; biomass)

There is a general concern, when setting specific goals at sectoral level, that the cost involved may be too different from the marginal cost incurred in other activities. Similarly, economists generally advise against setting technology-specific goals to reduce pollution on grounds that it can freeze innovation and also impose too high a cost on covered entities - there are exceptions, when toxicity is high and there is only one technology fix, which is not the case for GHG emissions. It is often preferred to express objectives in terms of overall performance, such as emissions per unit of output for a given process. This performance may, in the end, only be reachable with a specific technology, but technological innovation would be best encouraged if participants had the chance to be rewarded for going beyond the stated performance goal. On that basis, it is hard to argue that technology-specific should be preferred over performance-based goals; further, there should be incentives to go beyond the set performance objective.

\subsubsection{Pros and cons of technology-based approaches}

There are several possible advantages of technology-based approaches to GHG mitigation goals. These include:

- Visibility: a technology diffusion goal could be a very clear objective for possible donors, in case upfront finance were needed, or if technology transfer were required.

- Flexibility: technology-based crediting offers the opportunity for countries to design a technology target that suits their needs and is realistic in light of their technological know-how and access, or meet specific development needs.

- Simple monitoring: ensuring that countries have met a particular technology target could be relatively simple (e.g. surveying the numbers of installations in place and records of actual operation). Alternatively, if a technology-based approach was established for new plants only, planning permission

31 EU ETS and other cap-and-trade system development teach us that sectoral coverage need not be comprehensive. 
could require certain monitoring and reporting requirements. Simpler monitoring requirements would make reduced demands on the capacity of the host country to undertake them.

- Acceptability and risk:

- It has been argued that a technology target provides more certainty than an intensity target. For instance, lower than expected growth in a sector would slow down capital stock turnover and therefore inhibit performance improvement. A target to install $\mathrm{x}$ power plants with $\mathrm{y}$ technology may be more easily implemented, especially if financial support were guaranteed at the outset.

- Some developing countries, such as China, have expressed particular interest in a technologyoriented approach in a post-2012 climate regime. Technological innovation is a high political national priority. An international mechanism that supports the introduction of innovative technologies may therefore be more acceptable in the context of international climate negotiations.

The above points highlight some of the advantage of establishing clear technology goals for GHG mitigation, as a possible basis for international support. In some cases, a country could translate its technology objectives into intensity or fixed emission objectives, and agree to a "no lose" objective on such basis. Recent work (CCAP et al., 2008) shows the importance of the information on technology (including cost) to establish mitigation objectives that match the capacity of countries; again, the visibility of technology objectives may help countries to engage further on mitigation.

However, questions arise for an approach that would seek to credit GHG for technology performance beyond the initial goal. Turning now to the crediting aspects of technology approaches, some challenges must be addressed.

- How to establish the link between technology deployment and GHG mitigation, without a preagreed methodology to do so ${ }^{32}$ Agreeing to a methodology ex-post may be quite political, but also complicated by the knowledge of factors that affected the achievement of the goal. ${ }^{33}$

- Uncertain GHG impacts: the simpler the monitoring requirements, the more likely that estimates of GHG mitigation from a particular technology deployment will be uncertain. This is because the impact of technology deployment on GHG mitigation depends on how much installations are operated - not merely on the number of such installations. ${ }^{34}$ This would need to be taken into account at the outset in the monitoring requirements, else GHG mitigation would be hard to evaluate.

- How would the financial effort and possible benefits from credits be shared between possible donors for the acquisition of the technology and the recipient entities?

32 CDM methodologies offer some internationally-approved methods of calculating the GHG mitigation impact of particular actions linked to specific technology use. However, several of these methodologies needed to go through multiple iterations before they were approved - highlighting the fact that there can be different opinions of the most appropriate way of calculating GHG mitigation impacts.

33 What if, for instance, a GHG mitigation technology turned out to have witnessed a sharp decline in cost, or if the domestic support to a technology ended up being much more attractive than anticipated? Parties may then reconsider whether the agreed technology objective was indeed as ambitious as previously anticipated.

34 Alternatively, detailed monitoring requirements and methodologies for establishing the GHG equivalence of a particular technology deployment could be established. However, this would then create significant data requirements - which is counter to the whole idea behind technology-based crediting. 


\subsubsection{In summary}

Although there are downsides in adopting a technology prescriptive approach to GHG mitigation, a technology-based approach to GHG mitigation goals offers both potential advantages and disadvantages. Two fundamental issues need to be addressed if crediting were to play a role in such approaches.

The first relates to the estimation of associated emission reductions. The case of clean coal (or other specific technologies that lower or substitute fossil fuel use) illustrates the issues in moving from a technology goal to a crediting baseline. A target on the diffusion of efficient coal plants (X gigawatt of capacity by year $\mathrm{Y}$, or $\mathrm{Z} \%$ of capacity additions) would not be straightforwardly translated into a crediting level: the installation of $(X+10 \%)$ gigawatt of capacity with ultra-supercritical technology, does not in itself indicate the exact improvement over what would have happened otherwise, there would need to be a negotiation to define at which point credits could be issued, i.e. the baseline level. In light of the volumes that may be involved, leaving this question to be resolved after the agreement on the technology goal could be problematic. This raises another question on the nature of the technology goal: would it be voluntary, or negotiated among Parties?

The second issue relates to support. Could a country obtain financial support under the MRV clause of the Bali Action Plan, and also claim credits for its mitigation actions? This is conceivable as a package defined ex ante: a country agrees to a "no lose" sectoral target under the condition that specific know-how and technology be made available to it, or that support be granted to policy implementation. This may, however, be more controversial if a country asks to be credited ex post, when it has out-performed its technology goal. To what extent has the existing support made such achievement possible? Is it legitimate to generate credits if so?

More work needs to be done on the articulation between the two possible support mechanisms. Clearly, a country could define a crediting GHG baseline based on what it thinks a technology could deliver in terms of reductions. The baseline would be defined ex ante and the country would seek to outperform the intensity or fixed baseline with technology deployment as its main instrument. Alternatively, it could propose such policy for international support, without seeking credits.

\section{Moving Beyond Offsets}

Crediting emission reductions as it is known now (i.e. the Clean Development Mechanism) has been criticised, inter alia, for its high transaction cost which can discourage some potential projects; and the offsetting nature of the mechanism. The latter emphasises that while CDM lowers the overall cost of achieving the agreed environmental goal - the Kyoto targets of Annex B countries - it does not directly contribute to global emission reductions. As we illustrated in section 2, there is a related issue of possible oversupply. Shifting crediting mechanisms away from one-for-one offsets would address both these issues, as it would imply reducing overall potential supply (see EC, 2009; Chung, 2007; Waxman and Markey, 2009).

There are many options to increase the environmental contribution of crediting. These include at least three options on the supply side:

- Setting ambitious baselines, below business-as-usual (the principle underlying sectoral "no lose" targets, see e.g., Schmidt et al., 2008).

- Discounting emission credits issued by the various mechanisms: for one avoided tonne of $\mathrm{CO}_{2}$-eq below the baseline, less than one credit would be issued (e.g., Chung, 2007).

- Restricting the eligibility of sectors and activities for crediting, with mitigation to be encouraged by other means in other activities. This option would only be effective if other support mechanisms are effective in delivering mitigation. 
On the side of demand, three more options allow moving beyond offsetting:

- Discounting emission credits as they are used for compliance: an entity would need to surrender more than one credit for every ton of emissions being offset (e.g. Waxman-Markey draft, 2009).

- Acquiring and cancelling a portion of credits (e.g., Müller and Ghosh, 2008).

- The recognition rules of international credits in domestic carbon markets, and by individual governments on the buying side. These rules could include variations of several options like discounting on the demand side or eligibility of sectors/activities.

This section examines the pros and cons of the main options currently discussed, but first summarises some economic analysis of these options.

\subsection{An economic assessment of restrictions on the supply of GHG credits}

Schneider (2008) provides a ground-breaking analysis of the effects that discounting could have on CDM so-called CDM with atmospheric benefits. It discusses some pros and cons from theoretical and practical viewpoints. The first intuition of these effects may be that, generally speaking, discounting punishes project developers. The second is that the costs are borne by the developing country. The paper shows that neither of these intuitions may turn out to be true in realistic carbon market situations. The paper also highlights the further research needs and clearly highlights its limits.

The analysis begins with a simple illustration of where developed country mitigation would eventually occur, if choices were made on the basis of cost-effectiveness, and countries had full access to CDM projects in developing countries. The market would, in theory, lead to mitigation where it is less costly, to the point where the marginal cost in developed countries equals that of the developing countries. The price for CERs would then settle at that level (Figure 10).

Schneider (2008) adds two important assumptions:

- A constraint on how much developed countries can rely on CERs. This condition already applies in today's carbon markets, following EU decisions, and the supplementarity element of the Kyoto Protocol.

- A constant share of the emission reductions is no longer credited as CERs, hence contributing to lower global emissions.

The results (not illustrated in the figure) are essentially as follows:

- With limits on the use of the CDM by developed countries, the price of CERs is driven down, by a level of demand lower than would otherwise be the case. This reduces CDM-related income in CDM host countries.

- Reducing credits generated from CDM projects, e.g. by discounting, increases the price of CERs: an investor that is paid via discounted CERs will need a higher price per CER to conduct similar mitigation activities than at present.

Schneider concludes that while the import restrictions on CERs are at the expense of developing countries project developers, the discounting of CERs could roughly compensate for this by pushing prices up: "In $a$ carbon market with limitations on the use of the CDM, the CER suppliers benefit considerably from the introduction of a CDM with atmospheric benefits. In all three analysed cases (50\%, 60\% and 70\% crediting), the rents of the CER suppliers increase significantly compared to the situation without [...] atmospheric benefits." 
Schneider's theoretical result on both mechanisms compensating each other may have to be reviewed carefully, as it is based on an ideal market and derived from simple graphic representations of a linear CER supply in developing countries and abatement cost curves in developed countries. For instance, discounting credits (whether through a blanket discount rate or a negotiation of ambitious baselines) may have a different effect across mitigation activities. Markets have proven to be rather unpredictable in the past - see the unexpected development of HFC destruction projects and their effect on the supply CERs. Thus, assuming a linear response to discounting may be missing some important market adjustments.

Further, the paper assumes that the ex ante global mitigation objective, assigned to developed countries alone, is given, and independent from the discount rate that would apply to credits - a different discount rate would simply trigger more or less overall mitigation ex post. One could envision a different scenario in which Parties agree to a global mitigation, with the rate of discounting of CERs being part of the discussion, as a means to distribute the overall mitigation effort. In other words, the higher the "atmospheric benefits" of crediting, the less stringent the developed country goals ought to be, if we keep global mitigation constant.

Figure 10: The carbon market without limits on CDM use

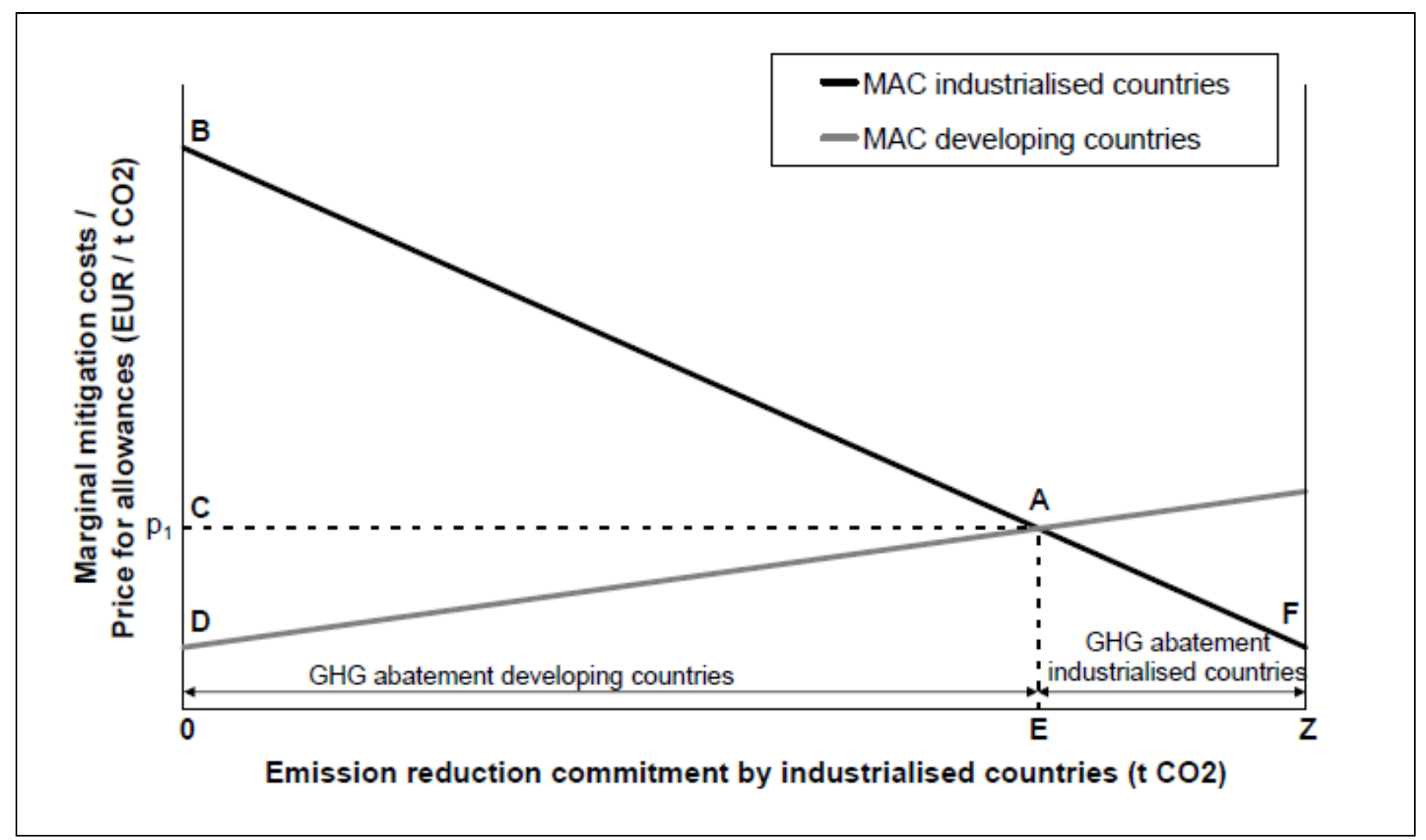

Source: Schneider, 2008

\subsection{Ambitious baselines}

An ambitious baseline for crediting is an essential element of the no-lose sectoral targets: it is because of the "no lose" nature of the objective that there could be an open discussion over an ambitious reduction goal. The absence of a penalty for non compliance seeks to reduce the risk attached with failure to meet the goal.

The ambitious baseline is also part of the discussion on countries' own contribution to, and responsibility for GHG mitigation activities; the definition of ambitious baselines may address the question of what are nationally-appropriate mitigation actions in these sectors, i.e. policy measures that can help move the baseline but do not necessarily require a carbon cost for implementation. In the absence of ambitious baselines, expanding crediting to whole sectors would mean increased subsidies for mitigation in 
developing countries, without a contribution to global reductions. This picture does not match the growing role of some emerging economies in the accumulation of GHG in the atmosphere, nor their economic and enhanced capacity to address this problem since the Rio agreement in 1992. There is, therefore, an effortsharing element that is an integral part of the discussion of how ambitious the baselines ought to be for various sectors and countries. The results of such a discussion would affect the countries potential revenues from a crediting mechanism, and would require a political agreement.

The baseline discussion cannot be entirely political, however. Countries need reasonably good data on their starting point and on their mitigation potential to get a grasp of what objective is feasible at what price, and of what type of support they may require. This is far from being a straightforward task in many countries and sectors, given current data constraints. Under Japan's proposal (Japan, 2009), emerging economies are asked to propose intensity targets for various industrial activities, a prerequisite for which is sound, recent data.

Efforts are underway (CCAP et al.; task-forces under APP; industry federations; IEA, 2008) to generate such data. Further, discussions of benchmarks for industry in the context of the EU Emissions Trading System should bring important methodological insights as well as reference points for any sectoral baseline discussion for crediting mechanisms. National circumstances would of course also play an important role in defining what is feasible in various activities.

\subsection{Discounting credits}

Discounting of credits has been discussed by Chung (2007) and Schneider (2008). Chung considers this option as an alternative to country-wide goals for developing countries: "We can generate a net global GHG emission reduction from developing countries (in an UNFCCC term, non-Annex 1 parties) without imposing targets on them, if we discount CERs generated from CDM projects". This paper generalises the discussion to sectoral objectives. ${ }^{35}$

Discounting is an option that can be applied both on the supply and the demand side. Schneider (2008), in his discussion of CDM with atmospheric benefits, argues that discounting on the supply side would be preferable: a fixed portion of calculated emission reductions would be issued as CERs. If applied on the demand side, there is a possibility that different countries that use CERs for compliance apply different discount rates, hereby creating market distortions with unpredictable effects, yet no obvious gain over the supply-side alternative.

However, the recognition rules in domestic carbon markets could imply discounting on the demand side: an emitter would have to hand in more than one credit per unit emitted. Such a discounting approach is one of the "use restrictions" that the Waxman-Markey draft bill proposed for the US system and which the revised EU ETS Directive enables to introduce in the third trading period. Yet, a precondition for demandside discounting to be effective is the coordination of recognition rules across linked carbon markets. Another case where demand-side discounting could be useful is in the event of a massive inflow of credits with questionable environmental quality; major buying markets could coordinate to discount units originated from these activities/countries.

While supply side discounting appears to be simpler, it may not be simpler to implement in practice. The discount rate of the credits requires a negotiation and the consent of those who lose from discounting, the credit suppliers - even if with restricted demand volume, Schneider shows that the overall effect may not be negative on sellers. Demand-side discounting also requires a negotiation of the discount rate, but may be simpler to achieve particularly if there is coordination of recognition rules across major carbon markets.

35 Alternatively, one could assume a much broader spectrum of activities being eligible for CDM. Another possible use for discounting under the CDM would be to organise the transition away from CDM for countries that would accede to a different level of effort. It may nevertheless be easier to let existing projects run their full crediting course... 
In theory, discounting and ambitious baselines can achieve the same result: a net contribution to global emission reductions, every time sector-wide efforts are undertaken. With a similar amount of net reductions generated by both approaches, the effect on the price of carbon would be identical. There is also the possibility that discount rates could be varied depending on host country categories, activities or projects. ${ }^{36}$

The key question to ask on discounting is whether it would simplify the process, compared to alternatives for sectoral crediting beyond offsets. Sector-wide baselines for crediting are likely to be both controversial and open to negotiation, be it simply because projections can be based on one of several different plausible future scenarios. Thus, sector-wide baselines are likely to differ according to a country's national circumstances, including its "common but differentiated responsibility and respective capability". It is then unclear why Parties would want to introduce an additional instrument, like a discount rate, to create further atmospheric benefits, when they can address this issue fully through the negotiation of baselines. ${ }^{37}$

\subsection{Retiring credits}

Müller and Ghosh (2008) propose to retire CERS as an approach to create fully measurable, reportable and verifiable actions (MRV), with support. A range of mitigation activities could go through a reformed CDM process, CERs would be issued and sold, but not all such CERs would be used by Parties for compliance. Parties could agree to a fund that would acquire credits and cancel them for that purpose.

The main difference between this and a no-lose sectoral target with an ambitious baseline is the requirement for mitigation activities (not just projects) to be channelled through the CDM process (sectoral CDM would work the same way, however). If the goal is to introduce wide-ranging policies and measures in developing countries, the CDM in its current form may be both too slow and too narrow. So this would imply a CDM reform for the sole purpose of allowing policies and measures to become eligible, with an eye to retire the issued CERs thereafter. The maybe simpler alternative would consist in setting proper measures to support nationally-appropriate mitigation actions.

\subsection{In summary}

The introduction of new forms of market mechanisms is motivated, in part, by the need to go beyond the offsetting nature of the existing project-based mechanism. As Schneider (2008) puts it, it is about generating atmospheric benefits that are additional to the reductions coming from countries that adopted binding emission caps. In other words, it is about the challenge to create acceptance in developing countries to move beyond the CDM-based offsetting approach and towards own mitigation contributions, until economy-wide objectives can be adopted. The questions posed here are: what mechanisms can trigger these additional environmental benefits, and what do they imply for the market? Will prices increase from what they would have been, with a continuation of the CDM? Will the total rent for countries on the supply side increase or decrease, given some already visible restrictions on the demand side?

Of the three options considered in detail - ambitious baselines, discounting credits, cancelling some credits - the ambitious baseline may be the most transparent and less cumbersome from a negotiation standpoint. Introducing a discount rate would open yet another political discussion, in addition to the discussion of

\footnotetext{
${ }^{36}$ Schneider provides a list of possible rationales for differentiated discounting: "favouring projects with large benefits for sustainable development [...] projects that use innovative technologies [...] Disfavouring projects that have very large windfall profits [...] projects with more questionable additionality”. He points out that discounting could also be used to influence the geographical balance of projects, or reflect various responsibility and capability to take action to mitigate climate change. The issue of vulnerability to international competition is also mentioned as a possible motivation for sectoral differentiation of credit discount rates.

37 There would also be an optics issue: a developed country would be free to trade AAUs at full value, while credits from developing countries, now with ambitious baselines, would be discounted... Discounting in this case would appear as a double penalty.
} 
ambition in the sectoral baseline. The cancellation of a portion of CDM credits, to be as broad-ranging as sectoral crediting, would require using the CDM as the sole conduit for quite diverse mitigation actions, from projects to sectoral targets - extensive CDM reform would be needed for this. Moreover, retiring credits - unless baselines were ambitious - would not imply an additional contribution by developing countries to global mitigation, as the resulting mitigation would still be paid by buyers, i.e. developed countries.

The expansion of crediting through sector-wide approaches is generally in favour of host countries, through a higher level of revenues. On the other hand, ambitious baselines, desirable from a global environmental perspective, would create a meaningful effort for developing countries, while addressing the issue of oversupply in a genuine way. The question of support, other than credits, may be of paramount importance in this discussion. Amongst all options, ambitious baselines also provide a natural link to ongoing discussions on own contributions from developing countries and on other types of support for low-cost mitigation activities.

\section{Conclusions}

The carbon market has shown that it can drive significant emission reductions including in developing countries - CDM could generate between 1.3 and $1.6 \mathrm{GtCO}_{2}$-eq of issued CERs by 2013; the CDM pipeline indicates a $7.6 \mathrm{GtCO}_{2}$-eq. of cumulative CERs by 2020 (UNFCCC, 2009; PointCarbon, 2009). Domestic and regional carbon markets have led to the formation of a carbon price that plays a vital role in driving investment in abatement. As such, the carbon market in general has a role to play in guiding further mitigation actions towards least-cost potentials.

Under the rubric of sectoral approaches, options for introducing sector-wide market mechanisms in developing countries could expand the carbon price signal, and reward investments in less GHG-intensive technologies and practices. These market mechanisms could be divided into two main categories: sectoral crediting mechanisms, where emissions below a baseline in a particular sector would generate ex post emission credits; or sectoral trading, where allowances are allocated on an ex ante basis.

From an economic perspective, this possible evolution in the carbon market is welcome. At the same time, it should occur in a way that meets expectations on the demand and supply sides, and reflects the environmental imperatives and political realities of global mitigation requirements. Our preliminary estimates show that the potential supply could be much larger than demand, depending on how quickly sectors and countries would establish sectoral objectives and achieve mitigation. As there may be hard limits on the demand side (as is already clear e.g. from the EU), some thought should be given to possible effects over time - a low price may deter needed innovation and contribute to a carbon lock-in that would hamper progress in following periods.

Some significant political and analytical investment is still needed to turn today's concepts of sectoral market mechanisms into reality; not all countries may be able, eligible, or required to undertake such actions; others, still, may consider the adoption of hard caps for sectors and participate in international emissions trading on such basis. Many uncertainties therefore remain on scope, political acceptability and the timing for the possible introduction of these mechanisms; but the carbon market has proven to be very effective in driving mitigation investment, which is why the move from projects to sectors must be well thought-out.

If crediting is to move to the sectoral scale, some have suggested to depart from the CDM practice of onefor-one emission reductions, to ensure that crediting should be accompanied with climate benefits, and not just economic and sustainable development benefits. In practice, this implies negotiating baselines that go beyond business-as-usual, while not introducing a penalty for not reaching such baselines, so as to minimise risk for countries that undertake such action. Greater participation in ambitious GHG mitigation goals may be encouraged by "no lose" goals than legally-binding objectives for which governments are 
liable in the case of non-compliance. It is not clear, however, whether both options would open to all developing country Parties in the 2013-2020 timeframe.

Regardless of any emission reduction goals and their legal nature, the elaboration of ambitious baselines requires data on current practice, some expertise on the available mitigation potential and cost, as well as some political negotiation over the effort that the host country is willing to make before crediting/trading starts - the sharing of the effort in global mitigation. For some designs of sectoral approaches (e.g. those using crediting baselines at a fixed emissions level), data could also be required on future activity levels. The process to arrive at such outcome is not defined yet, and options ought to be selected on the basis of their efficacy in getting the mechanism going quickly.

In contrast with intensity-based "no lose" crediting, ex ante allowance trading, if the government were to devolve allowances to entities, would enable these entities to manage this new asset and to pursue mitigation actions cost-effectively. The difficulty of allocating fixed emission goals to economic sectors for which international climate policy is so far synonymous with subsidies via potential CER revenues should not be underestimated, however. Depending on political will and capacity, intensity-based objectives may be more attractive, although not without technical difficulties.

Regarding technology-based sectoral objectives, they may present advantages in terms of political acceptability, and could indeed be used by countries to define sectoral emission objectives. However, unless the relationship between technology diffusion and GHG emission reductions is measurable, reportable and verifiable at the outset (thus making this approach very similar to a sectoral crediting mechanism) it is difficult to see how such objectives could provide access to the carbon market in a way that would make carbon finance a main driver for technology diffusion.

To be effective, sectoral market mechanisms should ideally relay the price signal directly to entities. Under one option envisaged here - crediting based on intensity targets, with performance measured in $\mathrm{CO}_{2}$ equivalent per unit of output - the price signal from the carbon market to these decision-makers is not direct because crediting is estimated at country level, based on aggregate sectoral performance. There are many options for a government to implement a sectoral goal at domestic level, with more or less direct access to the carbon market. These options rank from subsidies reform when they exist, mandated performance for new plants, to a plant-by-plant performance objective, the design of which may prove very cumbersome and data-intensive. The nature of the sector will also define what is most practical $-\mathrm{a}$ dimension that we have not explored here. ${ }^{38}$ In moving towards implementation, from negotiation on baselines/targets to actual domestic policy, a proper definition of coverage could prove to be strategic, e.g.: should all plants be included? What should be the threshold for participation? Should only new plants be considered, when sectors' emissions grow very rapidly, or would this create perverse incentives? Should effort focus on critical parts of a sector, not all of it? Such choices will define the extent of data collection needed to define baselines, as well as the number of installations to be governed by a sector-wide market instrument. These elements are also important for countries that will consider the administrative implications of moving down this path.

Last, Parties need to consider sectoral market mechanisms in an evolving international climate policy environment, and how the design of policy instruments for post-2012 mitigation could influence or constrain the choice of policy approaches later on. Today's uncertainty makes this task arduous. However, doing so would help to ensure an effective post-2012 framework.

38 In the context of REDD, Karousakis and Corfee-Morlot (2008) argues that carbon credits, because they would accrue to the government, would encourage them to remove subsidies that stand in the way of lower emissions. There may be cases, hence, where entities, even with the possibility to access the carbon market would still face perverse incentives. 


\section{Glossary}

AAU

AWG-KP

AWG-LCA

BAP

CDM

CER

$\mathrm{CO}_{2}$

Crediting

EU ETS

GDP

GHG

GIS

JI

NAMAs

$\mathrm{NO}_{\mathrm{X}}$

REDD

REDD+

Trading

SCM

$\mathrm{SO}_{2}$

UNFCCC

US EPA
An Assigned Amount Unit (AAU) is a tradable unit of $1 \mathrm{tCO}_{2}$ eq. The assigned amount is the total amount of GHG that each Annex B country is allowed to emit during the first commitment period of the Kyoto Protocol.

Ad Hoc Working Group on Further Commitments for Annex I Parties under the Kyoto Protocol

Ad Hoc Working Group on Long-term Cooperative Action under the Convention

Bali Action Plan

Clean Development Mechanism

A Certified Emission Reduction is the unit in which GHG emission reductions achieved by CDM are traded, and represents $1 \mathrm{tCO}_{2}$ eq.

Carbon dioxide

Crediting implies that trading units are issued ex-post after verification of performance

European Union Emissions Trading Scheme

Gross domestic product

Greenhouse gas

Green Investment Schemes

Joint Implementation

Nationally appropriate mitigation actions. Referred to in paragraph 1(b)(ii) of the Bali Action Plan, in relation to developing countries

Nitrogen oxides

Reducing emissions deforestation and forest degradation in developing countries

REDD+ covers deforestation and forest degradation, forest restoration, and forest activities leading to enhanced carbon storage

Trading implies that allowances are allocated ex-ante and can be traded at the beginning of the period

A Sectoral Crediting Mechanism credits emission reductions in a sector against a defined no-lose baseline

Sulfur dioxide

United Nations Framework Convention on Climate Change

United States Environmental Protection Agency 


\section{References}

Amatayakul, W., G. Berndes and J. Fenhann (2008), "Electricity sector no-lose targets in developing countries for post-2012: Assessment of emissions reduction and reduction credits", CD4CDM Working Paper No.6, December. UNEP Risoe centre.

APP Steel task force (2008), Status Review of Industry Indicators for Energy Saving, Task force Draft, November 2008, available at:

http://www.asiapacificpartnership.org/pdf/Steel/6th_meeting/Project2_Status_Review_of_Industry_I ndicators_for_Energy_Saving.pdf

Baron R., I. Barnsley and J. Ellis (2008), "Options for integrating sectoral approaches into the UNFCCC", OECD/IEA Information Paper. http://www.oecd.org/env/cc/41762381.pdf.

Baron, R. and Ellis, J. (2006), "Sectoral Crediting Mechanisms for Greenhouse Gas Mitigation: Institutional and Operational Issues", OECD/IEA Information Paper. http://www.oecd.org/env/cc/36737940.pdf

Carbon Trust (2009), Global Carbon Mechanisms: Emerging lessons and implications. The Carbon Trust 2009.

CCAP, CEPS, CCC, ZEW, IDDRI (2008), Sectoral Approaches: A Pathway to Nationally Appropriate Mitigation Actions. Center for Clean Air Policy Interim Report, Executive Overview, December 2008.

Chung, R. (2007), A CER discounting scheme could save climate change regime after 2012, Climate Policy 7(2007) 171-176.

Czech Republic (2009), A negotiation text for consideration at AWG-LCA 6, 28 April 2009. www.unfccc.int

Duan, Maosheng (2008), Clean Development Mechanism-Possible Ways for Scaling up, Presentation at IEA/IETA/EPRI workshop on emissions trading, http://www.iea.org/Textbase/work/2008/ghget/day2/Duan.pdf

EC (2009): Towards a comprehensive climate change agreement in Copenhagen. Communication from the Commission to the European Parliament, the Council, the European Economic and Social Committee and the Committee of the Regions. COM(2009) 39/3

Ellerman A.D. and B.K. Buchner (2008), Over-Allocation Or Abatement? A Preliminary Analysis of the EU ETS Based on the 2005-06 Emissions Data, Environmental and Resource Economics, Vol. 41, $\mathrm{N}^{\circ} 2,267-287$.

Ellis, J. and S. Moarif (2009), "GHG Mitigation Actions: MRV Issues and Options". OECD/IEA Climate Change Expert Group Papers, No. 2009/01, OECD Publishing. http://dx.doi.org/10.1787/5k4695890xd6-en

France (2008), Analysis of means to reach emission reduction targets and identification of ways to enhance their effectiveness and contribution to sustainable development: emissions trading and the projectbased mechanisms (AWG-KP). Further input in relation to the elements on possible improvements to emissions trading and the project-based mechanisms under the Kyoto Protocol. 5 November 2008. www.unfccc.int.

IEA (2009), Sectoral approaches - Building bridges to a safe climate. International Energy Agency, OECD, Paris. http://dx.doi.org/ 10.1787/9789264068735-en

IEA (2008), World Energy Outlook. International Energy Agency, OECD, Paris. http://dx.doi.org/10.1787/weo-2008-en 
IEA (2007), Tracking Industrial Energy Efficiency and $\mathrm{CO}_{2}$ Emissions. International Energy Agency, OECD, Paris. http://dx.doi.org/10.1787/9789264030404-en

Japan (2009), Submission of Japan for preparation of the Chair's document for the AWG-LCA5. UNFCCC

Karousakis, K. and J. Corfee-Morlot (2007), "Financing mechanisms to reduce emissions from deforestation: issues in design and implementation", OECD/IEA Information Paper. http://www.oecd.org/env/cc/39725582.pdf

Karousakis, K., B. Guay and C. Philibert (2008), "Differentiation Countries in Terms of Mitigation Commitments, Actions and Support". OECD/IEA Information Paper. http://www.oecd.org/env/cc/41762372.pdf

Kim, J.A., J. Corfee-Morlot and P. De T’Serclaes P. (2009), "Linking Mitigation Actions in Developing Countries with Mitigation Support: a Conceptual Framework". OECD/IEA Climate Change Expert Group Papers. No. 2009/01, OECD Publishing. http://dx.doi.org/10.1787/5k455r1485q4-en

Lewis, M.C. and I. Curien (2008), ETS Changes Approved by EU Parliament, Deutsche Bank Global Markets Research, 19 December 2008.

Müller B. and P. Ghosh (2008), Implementing the Bali Action Plan: What Role for the CDM? Oxford Energy and Environment Comment / Climate Strategies, October 2008.

NERA (2002), Evaluation of Alternative Initial Allocation Mechanisms in a European Union Greenhouse Gas Emissions Allowance Trading Scheme, prepared by David Harrison, Jr., and Daniel B. Radov, National Economic Research Associates.

New Carbon Finance (2009), "The impact of forestry on the global carbon market", Global Kyoto Research Note (25 February 2009).

New Carbon Finance (2009b), Waxman-Markley Proposal: Ambitious but with Concessions. North America - Analyst reaction (31 March 2009).

OECD (2008), The Economics of Climate Change Mitigation: Policies and Options for the Future. OECD, Paris. http://dx.doi.org/10.1787/228868001868

OECD (1992), Climate Change: Designing a Tradeable Permit System.OECD, Paris.

Ogonowski, M., N. Helme, D. Movius and J. Schmidt (2007), Reducing Emissions from Deforestation and Degradation: The Dual Markets Approach. Center for Clean Air Policy. August.

ONF International (2008), Reducing Emissions from Deforestation and Forest Degradation - Analysis of 7 outstanding issues for the inclusion of tropical forests in the international climate governance. Paris, France.

Schmidt, J., N. Helme, J. Lee, and M. Houdashelt (2008), Sector-Based Approach to the Post-2012 Climate Change Policy Architecture, Climate Policy 8 (2008), 494-515.

Schneider, L. (2008), A Clean Development Mechanism (CDM) with atmospheric benefits for a post-2012 climate regime. Öko-Institut e.V. Discussion Paper, 25 September.

Sterk W. (2008), From Clean Development Mechanism to Sectoral Crediting Approaches - Way forward or wrong turn?, JIKO Policy Paper 1/2008, Wuppertal Institute, Germany.

Ward M. (2008), Presentation at the IEA International Workshop on Sectoral Approaches to Climate Policy, 14-15 May 2008, www.iea.org

UNFCCC (2008), Zhejiang Guodian Beilun Ultra-supercritical Power Project (PDD), http://cdm.unfccc.int/UserManagement/FileStorage/TAQW906JVZIPFSKON2UMYHR7GB1843

US Congress (2009), H.R. 2454, The American Clean Energy and Security Act. http://energycommerce.house.gov/Press_111/20090515/hr2454.pdf 
Wertz-Kanounnikoff, S. (2008), Estimating the costs of reducing forest emissions. A review of methods. Center for International Forestry Research (CIFOR) Working Paper No. 42. 


\section{Appendix}

Table 3: Sectoral crediting illustration: revenues to government

\begin{tabular}{|c|c|c|}
\hline \multicolumn{3}{|c|}{ Revenues to government } \\
\hline & Country crediting baseline $\left(\mathrm{tCO}_{2} / \mathrm{MWh}\right)$ & 0.5 \\
\hline \multirow[t]{3}{*}{ Country total } & Output (1,000 MWh) & 100,000 \\
\hline & Average intensity $\left(\mathrm{tCO}_{2} / \mathrm{MWh}\right)$ & 0.455 \\
\hline & Emissions $\left(\mathrm{MtCO}_{2}\right)$ & 45.5 \\
\hline total $A+$ total $B$ & Total credits for sale on international market $\left(\mathrm{MtCO}_{2}\right)$ & 4.5 \\
\hline \multirow[t]{4}{*}{ Group A } & Output (1,000 MWh) & 50,000 \\
\hline & Intensity (tCO2/MWh) & 0.4 \\
\hline & Emissions $\left(\mathrm{MtCO}_{2}\right)$ & 20 \\
\hline & Emissions below baseline (negative: above) $\left(\mathrm{MtCO}_{2}\right)$ & 5.0 \\
\hline \multirow[t]{3}{*}{ Group B } & Output (1,000 MWh) & 50,000 \\
\hline & Intensity ( $\mathrm{tCO}_{2} / \mathrm{MWh}$ ) & 0.51 \\
\hline & Emissions $\left(\mathrm{MtCO}_{2}\right)$ & 25.5 \\
\hline total $B$ & Emissions below baseline (negative: above) $\left(\mathrm{MtCO}_{2}\right)$ & -0.5 \\
\hline
\end{tabular}

Table 4: Enhancing crediting certainty using a performance reserve

\begin{tabular}{|c|c|c|}
\hline \multicolumn{3}{|c|}{ Revenues to government } \\
\hline & $\begin{array}{l}\text { Country crediting baseline }\left(\mathrm{tCO}_{2} / \mathrm{MWh}\right) \\
\left.\text { Domestic baseline ( } \mathrm{tCO}_{2} / \mathrm{MWh}\right)\end{array}$ & $\begin{array}{r}0.5 \\
0.45\end{array}$ \\
\hline \multirow[t]{3}{*}{ Country total } & Output (1,000 MWh) & 100,000 \\
\hline & Average intensity $\left(\mathrm{tCO}_{2} / \mathrm{MWh}\right)$ & 0.455 \\
\hline & Emissions $\left(\mathrm{MtCO}_{2}\right)$ & 45.5 \\
\hline \multirow{2}{*}{ total $A+$ total $B$} & Total credits for sale on international market $\left(\mathrm{MtCO}_{2}\right)$ & 4.5 \\
\hline & Surplus credits to government $\left(\mathrm{MtCO}_{2}\right)$ & 2.0 \\
\hline \multirow[t]{3}{*}{ Group A } & Output (1,000 MWh) & 50,000 \\
\hline & Intensity (tCO2/MWh) & 0.4 \\
\hline & Emissions $\left(\mathrm{MtCO}_{2}\right)$ & 20 \\
\hline total $A$ & $\begin{array}{l}\text { Emissions below domestic baseline (negative: above) } \\
\left(\mathrm{MtCO}_{2}\right)\end{array}$ & 2.5 \\
\hline \multirow[t]{3}{*}{ Group B } & Output (1,000 MWh) & 50,000 \\
\hline & Intensity $\left(\mathrm{tCO}_{2} / \mathrm{MWh}\right)$ & 0.51 \\
\hline & Emissions $\left(\mathrm{MtCO}_{2}\right)$ & 25.5 \\
\hline total $B$ & Emissions below baseline (negative: above) $\left(\mathrm{MtCO}_{2}\right)$ & -3.0 \\
\hline
\end{tabular}




\section{www.oecd.org/env/cc/ccxg.htm}

\section{www.iea.org}

